\title{
The celestial mechanics approach: application to data of the GRACE mission
}

\author{
Gerhard Beutler · Adrian Jäggi · Leoš Mervart • \\ Ulrich Meyer
}

Received: 12 February 2010 / Accepted: 29 July 2010 / Published online: 21 August 2010

(C) Springer-Verlag 2010

\begin{abstract}
The celestial mechanics approach (CMA) has its roots in the Bernese GPS software and was extensively used for determining the orbits of high-orbiting satellites. The CMA was extended to determine the orbits of Low Earth Orbiting satellites (LEOs) equipped with GPS receivers and of constellations of LEOs equipped in addition with intersatellite links. In recent years the CMA was further developed and used for gravity field determination. The CMA was developed by the Astronomical Institute of the University of Bern (AIUB). The CMA is presented from the theoretical perspective in (Beutler et al. 2010). The key elements of the CMA are illustrated here using data from 50 days of GPS, K-Band, and accelerometer observations gathered by the Gravity Recovery And Climate Experiment (GRACE) mission in 2007. We study in particular the impact of (1) analyzing different observables [Global Positioning System (GPS) observations only, inter-satellite measurements only], (2) analyzing a combination of observations of different types on the level of the normal equation systems (NEQs), (3) using accelerometer data, (4) different orbit parametrizations (short-arc, reduced-dynamic) by imposing different constraints on the stochastic orbit parameters, and (5) using either the
\end{abstract}

G. Beutler $(\bowtie) \cdot$ A. Jäggi · U. Meyer

Astronomical Institute, University of Bern, Sidlerstrasse 5, 3012 Bern, Switzerland

e-mail: gerhard.beutler@aiub.unibe.ch

\section{Mervart}

Institute of Advanced Geodesy, Czech Technical University, K-152, FSvCVVT Thakurova 7, 16629 Prague 6-Dejvice,

Czech Republic inter-satellite ranges or their time derivatives. The so-called GRACE baseline, i.e., the achievable accuracy of the GRACE gravity field for a particular solution strategy, is established for the CMA.

Keywords Celestial mechanics - Orbit determination . Global gravity field modeling · CHAMP · GRACE-K-Band · GRACE accelerometers

\section{Introduction}

The celestial mechanics approach (CMA) has its roots in the Bernese GPS Software (Dach et al. 2007) and it is extensively used in this context since 1992 for determining the orbits of GNSS (Global Navigation Satellite Systems) by the Center for Orbit Determination in Europe (CODE) of the International GNSS Service (IGS). The CMA was generalized to determine the orbits of Low Earth Orbiting satellites (LEOs) and constellations of LEOs by Jäggi (2007) using the GPS observable. In recent years the CMA was extended to gravity field determination, including the use of highly accurate inter-satellite measurements.

The theoretical foundations of the CMA may be found in Beutler et al. (2010). Technical aspects like orbit integration, the efficient solution of the variational equations, etc., are outlined in that reference.

The present article has the focus on the special features of the CMA using GPS, K-Band, and, optionally, accelerometer data gathered by the GRACE mission in 2007. It is not the intention of this article to compare the solutions generated by the CMA to those generated by other groups. Such comparisons are, e.g., available in Prange et al. (2010) and Jäggi et al. (2010), where the emphasis is on particular 
solutions generated with the CMA, which have to be compared and/or validated using solutions generated by other groups. The results presented here are related to the options offered by the CMA and, to some extent, to the methods of celestial mechanics as such-applied to orbit and force field determination. This article is obliged to the Cartesian "discours de la méthode" (Descartes 1637).

Section 2 describes the data set analyzed, the a priori gravity field used and the gravity field to which the solutions are compared. The section introduces moreover the methods of comparing and validating solutions. Section 3 summarizes the characteristics of all solutions studied subsequently.

Section 4 deals with orbit determination of single satellites using spaceborne GPS and with orbit determination of close constellations using inter-satellite distance measurements in addition to GPS. The analysis scheme presented in this section is probably closely related to the one, which would be used by most analysts when tasked with precise orbit determination of a GRACE-like constellation of satellites. The results of this section are also used to define the mutual scaling of the GPS- and K-Band-specific normal equation systems (NEQs).

Section 5 introduces the standard analysis used so far to derive gravity fields with the CMA. CMA orbits are continuous by construction over the entire time span of one arc. The arc-length for analyzing data from the satellite missions is currently 1 day. The orbits are solutions of the deterministic equations of motion. By introducing instantaneous velocity changes (pulses) in the radial $(R)$, along-track $(S)$, and outof-plane $(W)$ directions or, alternatively, piecewise constant accelerations in these directions, the CMA orbits are given stochastic properties-for details we refer to Beutler et al. (2010). Section 6 studies the impact of solutions with free versus constrained pulses or accelerations.

The accelerometer measurements of the GRACE mission and their impact on gravity field estimation are studied in Sect. 7. All gravity field solutions discussed up to Sect. 7 are based on the K-Band range-rate observable. Alternative $\mathrm{K}$-Band observables, namely, ranges, range differences, and range double-differences, are studied in Sect. 8.

The K-Band residuals of range and range-rate solutions are analyzed in Sect. 9. From the theoretical point of view the K-Band range would be the primary observable-all other observables are derivatives of it. The standard range solutions presented in Sect. 8, however, cannot cope in quality with the best range-rate solutions. In Sect. 10 we show that range solutions of a quality comparable to the range-rate solutions can be generated with the CMA by making extensive use of the pseudo-stochastic parametrization.

The so-called GRACE-baseline (achievable accuracy of the gravity field with GRACE) is studied in Sect. 11 as a function of the CMA options. The major findings are summarized in Sect. 12.

\section{Observations, reference solutions, comparisons}

The GPS-derived kinematic satellite positions of the GRACE satellites, the K-Band measurements (ranges, range-differences, range-double-differences, range-rates) between them and, optionally, the accelerometer measurements of the mission are used for orbit and gravity field determination in this article. Data from days 50-99 of the year 2007 (DOY 50-99) are analyzed.

The gravity field AIUB-GRACE02Sp (Jäggi et al. 2009), estimated previously at the Astronomical Institute of the University of Bern (AIUB) with the CMA, is used as a priori information (and not modified for the orbit determination runs). The solutions generated in the following sections are compared to the solution AIUB-GRACE02S (Jäggi et al. 2010). The solution with the suffix "p" is based on the data from 2007, the solution without this suffix on data from 2006 and 2007. Apart from that the solutions were generated using the same pattern. The solution AIUB-GRACE02S

- is a biennial solution (with data from 2006 and 2007);

- was generated using the kinematic positions of GRACE-A and -B, the K-Band range-rates without taking the mathematical correlations into account, and the accelerometer measurements as empirically given (deterministic) functions (for a discussion of correlations of K-Band measurements we refer to Beutler et al. (2010));

- was generated with a fixed scaling ratio of $\sigma_{k b d, r r}^{2} / \sigma_{p h}^{2}=$ $10^{-10} \mathrm{~s}^{-2}$ between the daily K-Band- and GPS- NEQs;

- solved for all spherical harmonic coefficients between degrees $2 \leq n \leq 150$ without imposing any constraints or regularization on them;

- used the gravity field EGM96 (Lemoine et al. 1997) as the a priori field - and the solution was produced in one step;

- solved for piecewise constant accelerations in the three directions $R, S$ and $W$, set up at 15 min intervals;

- constrained the mean residual accelerations to $3 \times 10^{-9} \mathrm{~m} / \mathrm{s}^{2}$ in the $R-, S$-, and $W$-directions;

- used accelerometer data in the $R$ - and $S$ - directions, but ignored them in the $W$-direction (see Sect. 7);

- constrained the differences between simultaneous accelerations of the same type between GRACE-A and GRACE-B to $3 \times 10^{-11} \mathrm{~m} / \mathrm{s}^{2}$ for the $R$-, $S$-, and $W$ - directions.

The quality of the estimated gravity fields is assessed by the so-called difference degree amplitudes $\Delta a_{i}$, 
$\Delta a_{i}^{2} \doteq \sum_{k=0}^{i}\left[\left(C_{i k, \text { est }}-C_{i k, \text { ref }}\right)^{2}+\left(S_{i k, \text { est }}-S_{i k, \text { ref }}\right)^{2}\right]$,

where $i$ is the degree of the spherical harmonics coefficients, $k$ the order; "ref" stands for the reference, "est" for the estimated coefficients. In order to study the behavior of specific terms of the expansion the definition (1) is generalized in the following way:

$$
\begin{aligned}
\Delta a_{i ; l(i), u(i)}^{2} \doteq & \sum_{k=l(i)}^{u(i)}\left[\left(C_{i k, \text { est }}-C_{i k, \text { ref }}\right)^{2}\right. \\
& \left.+\left(S_{i k, \text { est }}-S_{i k, \text { ref }}\right)^{2}\right],
\end{aligned}
$$

where $l(i)$ and $u(i)$ are the lower and upper summation limits for each degree. For $l(i)=u(i)=0$ the behavior of the zonal terms is considered, for $l(i)=u(i)=i$ that of the sectorial terms. For $l(i)=0$ and $u(i)=i$ formula (2) reduces to the normal difference degree amplitudes, for $l(i)=i_{k}$ and $u(i)=i_{k}, i_{k} \neq 0$ the tesseral terms of a particular order are studied as a function of the degree.

The square roots of the formal error degree variances,

$\sigma_{i}^{2} \doteq \sum_{k=0}^{i}\left[\sigma^{2}\left(C_{i k, \text { est }}\right)+\sigma^{2}\left(S_{i k, \text { est }}\right)\right]$,

or briefly the error degree amplitudes, are used as a rough measure of the RMS error of the degree difference amplitude $\Delta a_{i}$ (covariances neglected). In analogy to Eqs. $(1,2)$ degree-specific limits will also be used in Eq. (3).

\section{Solutions performed}

The solutions generated here are based on the a priori gravity field AIUB-GRACE02Sp complete up to degree $n=150$, but determine only the gravity field up to degree $n=60$. Such a set-up might make sense to study monthly variations in the GRACE gravity field. This aspect is, however, not in the center of our interests. We use this option to generate many different solutions in an efficient way (concerning CPU time and storage requirements).

Table 1 lists all solutions made. The problem type (orbit or gravity field determination), the observable kind(s), the NEQ scaling ratio, the satellite(s), the use of the accelerometer data, the type of stochastic parameters, and the constraints imposed on the mean values and on the differences [definition see Beutler et al. 2010, Eq. (33)] of the orbit and stochastic parameters may be reconstructed from the solutions' names. The actual solutions are in addition time-tagged (year and day of the year for daily solutions, DOY/2007 for first and last day included for gravity field determination). When applying the constraints, the value $\sigma_{0}$ of the weight unit must be

\begin{tabular}{|c|c|}
\hline Label & Solution characteristics \\
\hline \multirow{4}{*}{$\begin{array}{l}\mathrm{PTT}(\mathrm{GK}) \mathrm{ASmm} S m S d \\
\mathrm{P}\end{array}$} & General problem characterization \\
\hline & Problem type \\
\hline & $\mathrm{P} \doteq \mathrm{O}:$ Orbit determination \\
\hline & $\mathrm{P} \doteq \mathrm{G}$ : Gravity field determination \\
\hline \multirow[t]{8}{*}{ TT } & Observables \\
\hline & GA: GPS-derived positions, GRACE-A \\
\hline & GB: GPS-derived positions, GRACE-B \\
\hline & GC: GPS-derived positions, GRACE-A and -B \\
\hline & RG: GPS-derived positions and K-Band ranges \\
\hline & $\begin{array}{l}\text { RD: GPS-derived positions and K-Band range- } \\
\text { differences }\end{array}$ \\
\hline & $\begin{array}{l}\text { DD: GPS-derived positions and K-Band range- } \\
\text { double-differences }\end{array}$ \\
\hline & $\begin{array}{l}\text { RR: GPS-derived positions and K-Band range- } \\
\text { rates }\end{array}$ \\
\hline \multirow[t]{3}{*}{ GK } & $\begin{array}{l}\text { Ratio } \sigma_{k b d}: \sigma_{p h} \text { (K-Band : GPS) for NEQ } \\
\text { constituents; }\end{array}$ \\
\hline & Only for types $\mathrm{TT}=\mathrm{RG}, \mathrm{RD}, \mathrm{RR}$ \\
\hline & $\mathrm{GK}=16: \sigma_{k b d}: \sigma_{p h}=1 \times 10^{-6}($ example $)$ \\
\hline \multirow[t]{3}{*}{ A } & Treatment of accelerometer measurements \\
\hline & $\mathrm{A} \doteq \mathrm{U}:$ Accelerometer measurements Used \\
\hline & $\mathrm{A} \doteq \mathrm{N}$ : Accelerometer measurements Not used \\
\hline \multirow[t]{2}{*}{$S$} & Type of stochastic parameters \\
\hline & $\mathrm{S} \doteq \mathrm{P}:$ Pulses \\
\hline \multirow[t]{2}{*}{$\mathrm{mm}$} & Spacing between stochastic pulses ( min) \\
\hline & $\begin{array}{l}\mathrm{mm}=15: \text { Spacing between subsequent sto- } \\
\text { chastic epochs } 15 \mathrm{~min}\end{array}$ \\
\hline \multirow[t]{2}{*}{$S m$} & $\begin{array}{l}\sigma_{\text {abs }} \text { of stochastic parameter for mean stochas- } \\
\text { tic parameters }\end{array}$ \\
\hline & $S m=34: \sigma_{\mathrm{abs}}=3 \times 10^{-4}$ \\
\hline \multirow[t]{2}{*}{$S d$} & $\begin{array}{l}\sigma_{\text {rel }}=\sigma_{\text {abs }} S \times 10^{-d} \text { for relative stochastic } \\
\text { parameters }\end{array}$ \\
\hline & $S d=12: \sigma_{\mathrm{rel}}=\sigma_{\mathrm{abs}} \times 10^{-2}$ \\
\hline
\end{tabular}

Table 1 Solution with label PTT(GK)SmmSm Sd

specified. For GPS-only solutions we use $\sigma_{0}=2 \mathrm{~mm}$ for the L1 (and L2) phase measurements, when analyzing K-Band range-rates or a combination of GPS and range-rates $\sigma_{0}=$ $0.2 \mu \mathrm{m} / \mathrm{s}$. Other combinations of observables are considered in Sect. 8. The corresponding values for $\sigma_{0}$ are specified in Eq. (12).

Table 2 contains the list of solutions (without time tags) actually analyzed in each section.

The experiments listed in Table 2 were usually compared to the biennial solution AIUB-GRACE02. One should keep in mind that this solution does not contain time variations of the gravity field. Our results thus might be obstructed by this neglect. But as we usually compare two or more solutions, and are in principle only interested in the mutual 
Table 2 Solutions performed

\begin{tabular}{|c|c|c|}
\hline Label & Section & Comments \\
\hline OGXUP15 & 4 & $\begin{array}{l}\text { Orbit det. using kinematic positions of GRACE-X, } X \in\{A, B, C\} \text {, } \\
\text { pulses with } 15 \text { min spacing, no constraints, accelerometers used. }\end{array}$ \\
\hline ORRxxUP15 & 4 & $\begin{array}{l}\text { Orbit det. using only K-Band range-rates pulses with } 15 \text { min spacing, } \\
\text { weak constraints, accelerometers used. }\end{array}$ \\
\hline GGCUPmm & 5 & $\begin{array}{l}\text { Gravity field det. using kinematic positions, pulses with spacing of } \mathrm{mm} \\
\mathrm{min}, \mathrm{mm} \in\{5,15,30\} \mathrm{min} \text {, with accelerometers, no constraints for } \\
30 \text { min, and } 15 \text { min solutions, weak constraints for } 5 \text { min solutions. }\end{array}$ \\
\hline GGCUP053810 & 5 & $\begin{array}{l}\text { Gravity field det. using only GPS (GRACE-A and GRACE-B), pulses } \\
\text { with } 15 \text { min spacing, with accelerometers, weak constraints. }\end{array}$ \\
\hline GRRxxUPmm & 5 & $\begin{array}{l}\text { Gravity field det. using only K-Band range-rates pulses with } \mathrm{mm} \text { min } \\
\text { spacing, } \mathrm{mm} \in\{5,15,30\} \text { min, with accelerometers, weak constraints. }\end{array}$ \\
\hline GRR14UPmm & 5 & $\begin{array}{l}\text { Gravity field det. using GPS and K-Band range-rates } \sigma_{K B D, r r} / \sigma_{p h}= \\
1 \times 10^{-4} \text {, pulses with spacing of mm min, } \operatorname{mm} \in\{5,15,30\} \text { min, with } \\
\text { accelerometers, weak/no/no constraints. }\end{array}$ \\
\hline GRR14UP051610 & 6 & $\begin{array}{l}\text { Gravity field det. using K-Band range-rates and GPS, pulses with } 5 \text { min } \\
\text { spacing, with accelerometers, constraints } \sigma_{v_{l}}=1 \times 10^{-6} \mathrm{~m} / \mathrm{s} \text { for mean } \\
\text { values and for half differences of pulses. }\end{array}$ \\
\hline GRR14UP051612 & 6 & $\begin{array}{l}\text { Gravity field det. using only K-Band range-rates, pulses with } 5 \text { min } \\
\text { spacing, with accelerometers, constraints } \sigma_{v_{l}}=1 \times 10^{-6} \mathrm{~m} / \mathrm{s} \text { for mean } \\
\text { values and } \sigma_{v_{l}}=1 \times 10^{-8} \mathrm{~m} / \mathrm{s} \text { for half differences of pulses. }\end{array}$ \\
\hline GGCNPmm & 7 & $\begin{array}{l}\text { Gravity field det. using only GPS (GRACE-A and GRACE-B), pulses, } \\
\text { without accelerometers, pulses with spacing of } \mathrm{mm} \mathrm{min,} \mathrm{mm} \in \\
\{5,15,30\} \mathrm{min} \text {, weak/no/no constraints. }\end{array}$ \\
\hline GRR14NPmm & 7 & $\begin{array}{l}\text { Gravity field det. using GPS and K-Band range-rates, pulses with spac- } \\
\text { ing of } \mathrm{mm} \min , \operatorname{mm} \in\{5,15,30\} \mathrm{min} \text {, with accelerometers, weak/no/no } \\
\text { constraints. }\end{array}$ \\
\hline GRR14NP051612 & 7 & $\begin{array}{l}\text { Gravity field det. using GPS and K-Band range-rates, pulses with spac- } \\
\text { ing of } \Delta t_{p}=5 \mathrm{~min} \text {, without accelerometers, constraints } \sigma_{v_{l}}=1 \times \\
10^{-6} \mathrm{~m} / \mathrm{s} \text { for mean values and } \sigma_{v_{l}}=1 \times 10^{-8} \mathrm{~m} / \mathrm{s} \text { for half differences } \\
\text { of pulses. }\end{array}$ \\
\hline GRD54UP30 & 8 & $\begin{array}{l}\text { Gravity field det. using GPS and K-Band range differences, } \\
\sigma_{K B D, r d} / \sigma_{p h}=5 \times 10^{-4} \text {, pulses with } 30 \text { min spacing, accelerometer } \\
\text { data used, no constraints. }\end{array}$ \\
\hline GDD84UP30 & 8 & $\begin{array}{l}\text { Gravity field det. using GPS and K-Band range-double-differences, } \\
\sigma_{K B D, r d d} / \sigma_{p h}=8 \times 10^{-4} \text {, pulses with } 30 \mathrm{~min} \text { spacing, accelerom- } \\
\text { eter data used, no constraints. }\end{array}$ \\
\hline GRG34UP30 & 8 & $\begin{array}{l}\text { Gravity field det. using GPS and K-Band ranges without correlations, } \\
\sigma_{K B D, r} / \sigma_{p h}=3.46 \times 10^{-4} \text {, pulses with } 30 \mathrm{~min} \text { spacing, accelerometer } \\
\text { data used, no constraints. }\end{array}$ \\
\hline GRG33UP30 & 8 & $\begin{array}{l}\text { Gravity field det. using GPS and K-Band ranges with correlations, } \\
\sigma_{K B D, r, 1 a} / \sigma_{p h}=2.53 \times 10^{-3} \text {, pulses with } 30 \text { min spacing, accel- } \\
\text { erometer data used, no constraints. }\end{array}$ \\
\hline
\end{tabular}

performance of them, this neglect does not affect our main findings.

\section{Standard processing for orbit determination}

Orbits are determined separately for GRACE-A and GRACE-B using only the GPS-derived kinematic positions as pseudo-observations. Solutions OGAUP15, OGBUP15, OGAUA1539 (see Table 2), are solutions of this kind. No attempt was made here to improve the GPS-derived position differences between GRACE-A and GRACE-B, e.g., by solving for the initial phase ambiguities of the GPS phase difference observations as recorded by the GRACE-A and -B receivers. Experiments of this type were performed by Jäggi (2007).
Orbits for the constellation may be determined using the kinematic positions and the K-Band observations. In the experiments performed in this section the accelerometer measurements were introduced as empirically given and the following accelerometer-specific parameters were determined:

- $\quad$ one offset parameter per day in the directions $R$ and $W$,

- a polynomial of degree $q=3$ in the $S$-direction to absorb slow time variations of the bias parameter,

- once-per-revolution terms in $R, S$, and $W$.

It is in principle possible to solve for a scale parameter. This option was not used subsequently (mainly due to strong correlations with the once-per-rev parameters).

It is not possible to determine the orbits of the two satellites by analyzing only K-Band data and to solve for all 


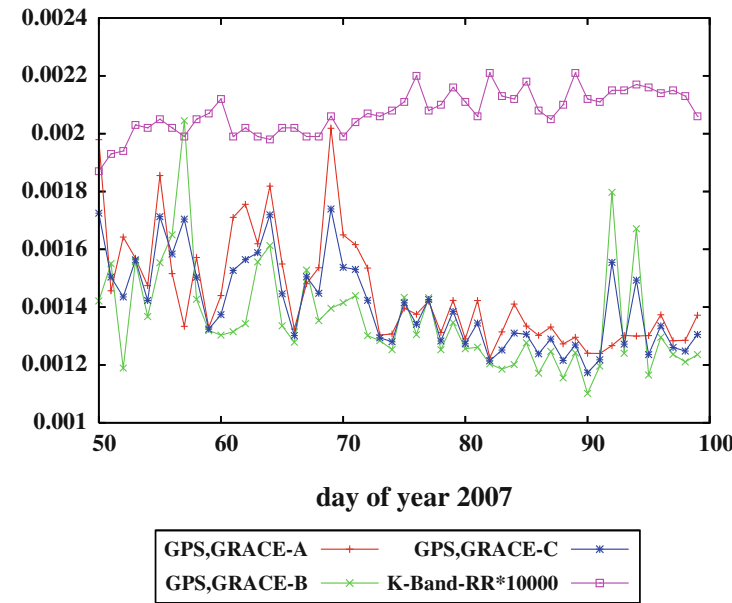

Fig. 1 Left: RMS error of orbit determination using GRACE-A kinematic positions (m), GRACE-B kinematic positions (m), GRACE-A and GRACE-B kinematic positions $(\mathrm{m})$, and K-Band range-rates $(\mathrm{m} / \mathrm{s}$,

orbit parameters pertaining to the two satellites-the corresponding NEQ-matrix would become singular. A non-singular differential orbit determination problem using only the $\mathrm{K}$-Band observations is defined by solving only for the differences $\mathbf{p}_{2}$ [Beutler et al. 2010, Eqs. (33)] of the orbit and stochastic parameters pertaining to the satellites, GRACEA and GRACE-B, and by slightly constraining the differences of the corresponding orbit parameters to the a priori GPS-derived orbit parameters. This scheme makes sense, because the K-Band observations are only very weakly dependent on the mean values $\mathbf{p}_{1}$ of the orbit parameters and because they are almost uniquely sensitive to the $S$-direction (see Eqs. (33)). The K-Band-only solution bears the label ORRxxUA153912, "xx" standing for a zero weight on the GPS NEQs. The square of the ratio of the GPSand K-band-only RMS values a posteriori may subsequently serve as the weight factor when combining GPS- and K-Band NEQs.

Figure 1 (left) shows the RMS errors a posteriori [GPS L1 phase observable, see Beutler et al. 2010, Sect. 3.2] of the daily orbit determination steps OGAUP15, OGBUP15, OGCUP15, and ORRxxUP15 (the latter multiplied by a factor of $10^{4}$ ) as a function of the day of the year. The RMS errors of the GPS-only solutions for GRACE-A and GRACE$B$ reveal essentially the same pattern as a function of time as the two satellites have almost identical properties, are in nearly the same orbital plane and observe simultaneously the same GPS satellites. Figure 1 (left) shows in addition the RMS of the GPS-only solutions using the GRACE-A and GRACE-B GPS-data together (solution OGCUP15, label GRACE-C). The latter RMS errors were used as $\sigma_{p h}$ to derive the ratio $\sigma_{k b d}^{2} / \sigma_{p h}^{2}$ for each day.

Figure 1 (right) shows the day-specific ratios $\sigma_{k b d}^{2} / \sigma_{p h}^{2}$ for the GPS- and K-Band-specific NEQ contributions derived
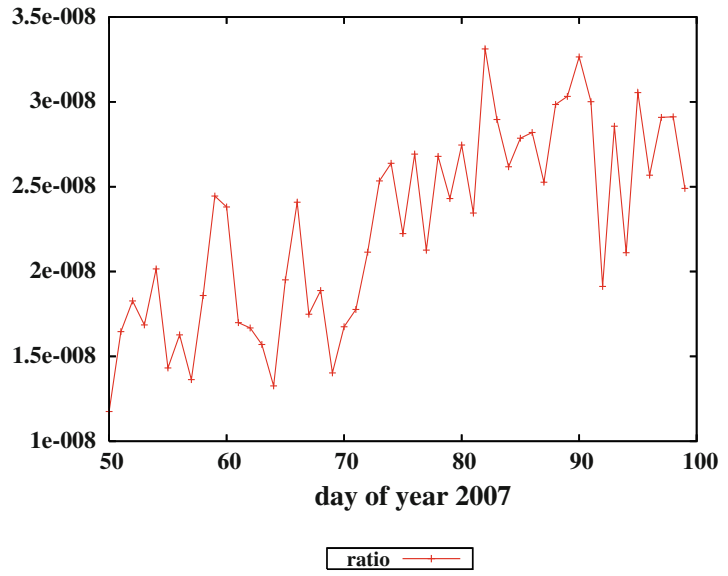

multiplied by $10^{4}$ ). Solutions OGAUP15yyddd, OGBUP15yyddd, OGCUP15yyddd, ORRUP15yyddd; right: Derived ratio $\sigma_{k b d}^{2} / \sigma_{g p s}^{2}$ using GRACE-C

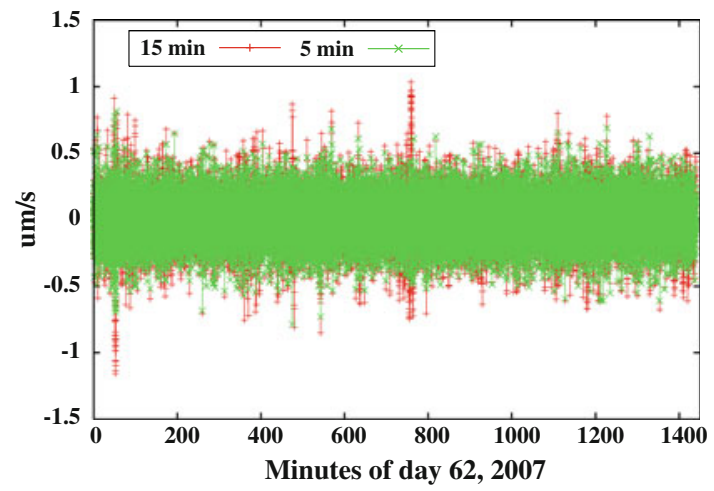

Fig. 2 Residuals of K-Band range-rate observations $(\mu \mathrm{m} / \mathrm{s})$ using a pulse spacing of 15 and $5 \mathrm{~min}$, respectively, DOY 62

from the RMS errors of solutions OGCUP15yyddd and ORRxxUPyyddd in Fig. 1 (left). These values are used when analyzing GPS- and K-Band range-rate data together and parametrizing the orbits with pulses (spacing of $15 \mathrm{~min}$, no constraints). Figure 2 shows that the pattern of the range-rate residuals and the corresponding RMS error depends somewhat on the spacing of the pulses. The systematic effects still present in the solution with a spacing of $15 \mathrm{~min}$ obviously can be absorbed to some extent by reducing the spacing from 15 to 5 min.

As the orbit and gravity field determinations are not very sensitive to small variations of the weight ratio within, let us say, a factor of 3, a general ratio of $\sigma_{p h}^{2} / \sigma_{p h}^{2} \doteq 10^{-8} \mathrm{~s}^{-2}$ will be used subsequently. Note that this ratio does not give the best consistency of our gravity fields with those of other GRACE analysis teams. Values of the order of $\sigma_{k b d}^{2} / \sigma_{p h}^{2} \approx$ $10^{-10} \mathrm{~s}^{-2}$, in essence de-weighting the GPS-contributions, make our results more consistent to those of other groups. 
This issue is, however, not of interest for the subsequent experiments.

Figures 1 and 2 document the simplest analysis scheme in orbit (and gravity field) determination, the short-arc scheme: The daily arc is subdivided into contiguous short arcs of $\Delta t_{p} \min$, where $\Delta t_{p}=15 \mathrm{~min}$ in the example. A similar experiment was performed by replacing the pulses by piecewise constant accelerations with the same spacing as the pulses. Slightly smaller ratios of about $\sigma_{k b d}^{2} / \sigma_{p h}^{2} \approx$ $(0.5-1.5) \times 10^{-8} \mathrm{~s}^{-2}$ could be achieved.

\section{Standard processing for gravity field determination}

Gravity field analysis may either be based on the GPS alone or on a combination of the GPS and the K-Band observables. Although this is not really correct, one may also use only the K-Band NEQs to derive a gravity field in analogy to the differential orbit determination strategy introduced in Sect. 4. Solutions of this kind will be generated here in order to validate the K-Band-only and the GPS-only contributions of the estimated gravity fields. As in Sect. 4 the accelerometer measurements are used as empirically given deterministic values. The same accelerometer-specific parameters are determined as in Sect. 4.

In this section the CMA is used to determine the spherical harmonics coefficients between the degrees $2 \leq i \leq 60$. The terms of degrees $61 \leq i \leq 150$ are taken over and kept fixed from the solution AIUB-GRACE02Sp. The coefficients of degree $n>150$ are set to zero. No constraints or regularizations are put on the estimated gravity field parameters. The observations of DOY 50-99/2007 are used in this section-as in Sect. 4.

Gravity fields determined only by the kinematic positions are documented in Fig. 3. All solutions are based on pulses in $R, S$, and $W$ with a spacing of 5,15 and $30 \mathrm{~min}$, respectively. The solutions are compared to the biennial solution AIUB-GRACE02S using Eq. (1). For reference purpose the solution EIGEN-05S (Förste et al. 2008) is included, as well. The pulses are unconstrained for the solutions based on the 15 and $30 \mathrm{~min}$ intervals. It was necessary to put weak constraints of $10^{-4} \mathrm{~m} / \mathrm{s}$ on the $5 \mathrm{~min}$ solution to avoid true singularities due to data outages, because a linear dependency is introduced if two pulses are set up at times $t_{a}$ and $t_{b}$ and if there are no kinematic positions in the interval $\left[t_{a}, t_{b}\right]$. The constraints imposed on the $5 \mathrm{~min}$ pulses are weak enough not to influence the resulting gravity field significantly. The solid lines in Fig. 3 show the difference degree amplitudes of the three short-arc solutions w.r.t. the biennial solution AIUBGRACE02S. The quality of the three solutions is almost the same between the degrees $15<i<50$. The lower degrees agree better with AIUB-GRACE02S for a wider spacing of

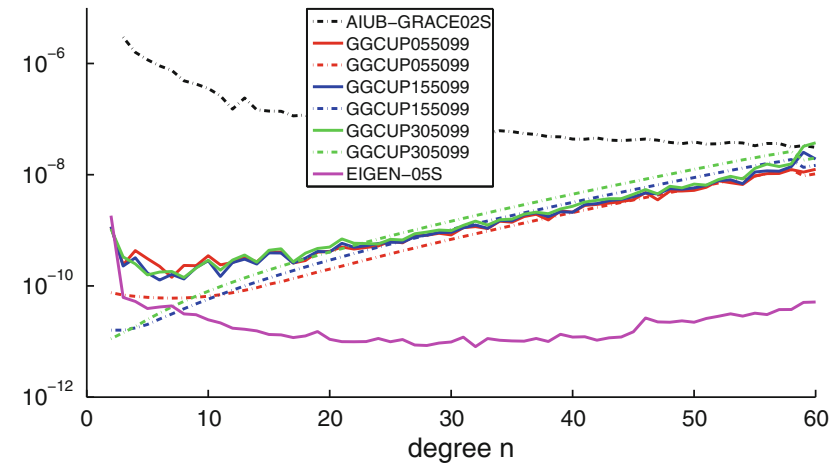

Fig. 3 Difference degree amplitudes w.r.t. AIUB-GRACE02S (solid) and error degree amplitudes (dash-dot) of GPS-only gravity fields based on short-arcs of 5, 15, and 30 min length; EIGEN-05S difference degree amplitudes w.r.t. AIUB-GRACE02S included for reference

the pulses, for the degrees $n>50$ the opposite is true. The dash-dot lines in Fig. 3, showing the error degree amplitudes according to Eq. (3), explain the quality patterns of the three solutions for the low degree terms: setting up more and more unconstrained or only weakly constrained pulses does not allow it to the parameter estimation process to distinguish between the long-wave gravity field terms and a long wavelength signal, which may be absorbed by the pulses. This has to be expected, because signals with periods $P>\Delta t_{p}$ may very well be absorbed by the pulses. One might ask the question whether the offset between the three curves is significant. Both answers, positive and negative, may be given: The dash-dot curves in Fig. 3 are computed from the RMS errors a posteriori, i.e., they reflect the product of the estimated mean error a posteriori of the observations and the square roots of the diagonal elements of the corresponding cofactor matrix, the inverse NEQ matrix. In a covariance study based on the white noise assumption one would replace the three RMS errors a posteriori by one and the same RMS error a priori. In this case the three error degree amplitudes would practically coincide in the interval of degrees $15<n<50$. In the presence of biases in the kinematic positions more and more of the low-frequency systematics may be absorbed by the pulses, implying that the mean errors a posteriori decrease with decreasing pulse separation $\Delta t_{p}(1.89,1.36,0.89 \mathrm{~mm}$ for $\Delta t_{p}=30,15,5 \mathrm{~min}$ in our case) - and the three error degree amplitude curves should be different. The "abnormal" behavior of the error degree variances for the degrees 59 and 60 is caused by the cut-off degree $n=60$ in the estimation process.

Figure 4 shows that GPS determines the sectorial terms of the gravity field much better than the zonal terms, because the sectorial terms generate long-wave perturbations along a polar orbit. Figure 4 shows that the RMS errors of the gravity field coefficients drop by 1-3 order of magnitude from the zonal to the sectorial terms. This fact indicates that the 


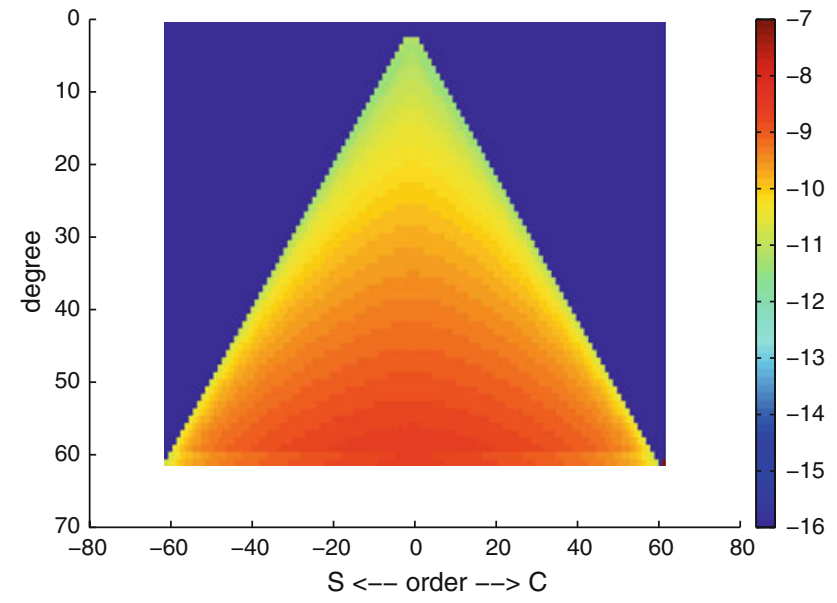

Fig. 4 Formal RMS errors of spherical harmonics coefficients (logarithmic scale) of solution GGCUP15

difference degree amplitudes and the corresponding error degree amplitudes in Fig. 3 usually are dominated by the error amplitudes in the (close to) zonal terms.

Instead of introducing and solving for the two satellitespecific orbit and stochastic parameter sets $\mathbf{o}_{1}$ and $\mathbf{o}_{1}$ their mean values $\mathbf{p}_{1}=\left(\mathbf{o}_{1}+\mathbf{o}_{2}\right) / 2$ and half differences $\mathbf{p}_{2}=\left(\mathbf{o}_{1}-\mathbf{o}_{2}\right) / 2$ are used as parameters in the CMA [Beutler et al. 2010, Eq. (33)]. This parameter transformation has several advantages. Here we make use of the fact that $\mathbf{p}_{1}$ is almost uniquely determined by the GPS observations, whereas $\mathbf{p}_{2}$ is mainly determined by the K-Band observations. Therefore, the mean values $\mathbf{p}_{1}$ were left out (i.e., kept fixed on the a priori values determined by GPS) from the full set of orbit and stochastic parameters for the K-Band-only solution. Weak constraints were put on the parameters $\mathbf{p}_{2}$, i.e., on the pulse differences and on the differences of the orbit elements. The following constraints were applied on half the differences of the initial osculating elements and of the pulses: $\sigma_{a}=1 \mathrm{~m}, \sigma_{e}=1 \times 10^{-3}, \sigma_{i}=\sigma_{\Omega}=\sigma_{\omega}=$ $\sigma_{u}=10 \operatorname{arcsec}, \sigma_{R}=\sigma_{S}=\sigma_{W}=10^{-3} \mathrm{~m} / \mathrm{s}$. The symbols $a, e, i, \Omega, \omega, u$ stand for the semi-major axis, the numerical eccentricity, the inclination of the orbital plane w.r.t. the equatorial plane, the right ascension of the ascending node, the angular distance of the perigee from the ascending node, and for the argument of latitude, respectively; the symbols $R, S$, and $W$ stand for velocity changes (pulses) in the radial, along-track, and out of plane directions. All elements refer to the initial epoch. For more details please consult Beutler et al. (2010).

The solid lines in Fig. 5 show the degree difference amplitudes of the solutions with 5,15 and $30 \mathrm{~min}$ pulse spacing w.r.t. the solution AIUB-GRACE02S, the dash-dot lines show the corresponding error degree amplitudes. The three solutions obviously are highly consistent with AIUBGRACE02S. The consistency increases with the pulse

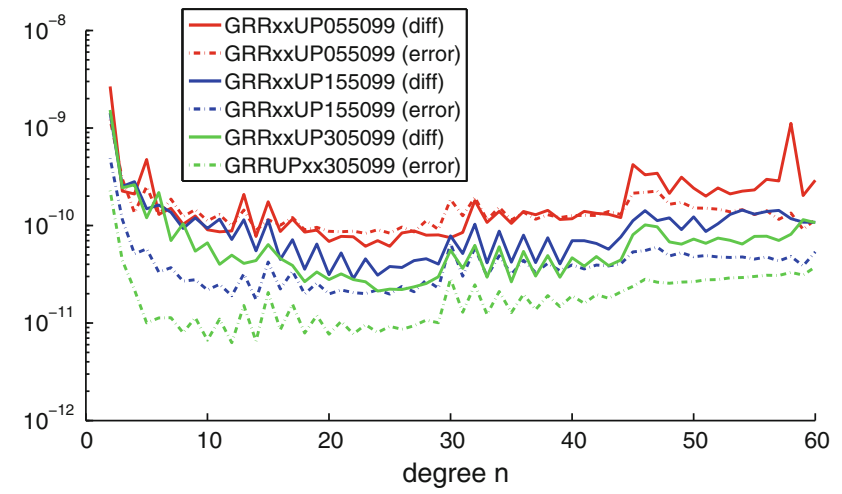

Fig. 5 Difference degree amplitudes w.r.t. AIUB-GRACE02S (solid) and error degree amplitudes (dash-dot) of range-rate-only gravity fields based on short-arcs of 5, 15, and 30 min length

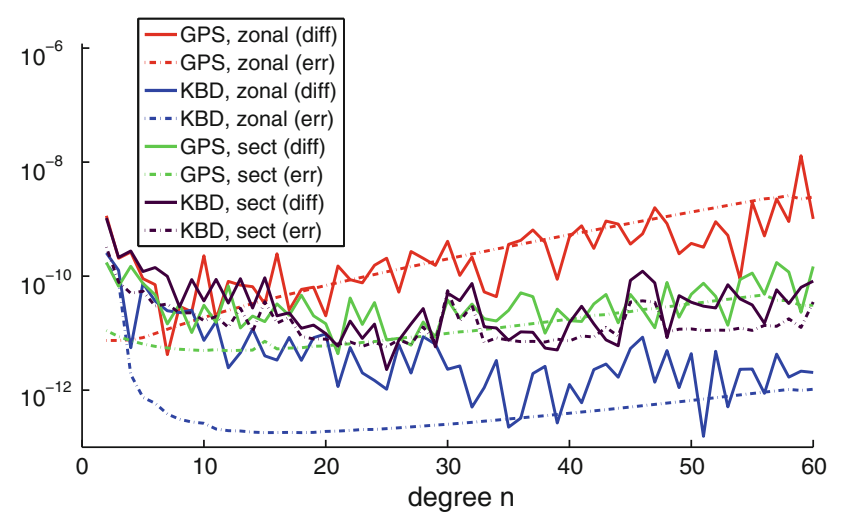

Fig. 6 Difference degree amplitudes w.r.t. AIUB-GRACE02S (solid) and error degree amplitudes (dash-dot) of GPS-only (zonal: red, sectorial: green) and K-Band (range-rate)-only (blue zonal, black sectorial) coefficients based on short-arcs of 15 min length

spacing. The error degree amplitudes are in turn roughly consistent with the degree difference amplitudes.

A comparison of Figs. 3 and 5 shows that K-Band has a much higher potential to contribute to a combined gravity field and that the combined gravity field in general is dominated by the K-Band contribution. The contribution is, however, substantially different for different types of harmonic coefficients. Figure 6 illustrates this fact for the $15 \mathrm{~min}$ short-arc solutions: The solid lines of Fig. 6 (red: GPS, blue: $\mathrm{K}$-Band, range-rate) show the degree differences of the zonal terms w.r.t. AIUB-GRACE02S, the dash-dot-lines of the same color the corresponding error degree amplitudes. The green and black lines in Fig. 6 give the same information for the sectorial terms, where green corresponds to GPS, black to K-Band. GPS obviously cannot contribute much to the zonal terms above degree $n \approx 5$. It has, however, the potential to contribute up to degree $n \approx 60$ (and beyond) to the determination of the sectorial terms.

Figure 6 also tells that it is difficult to assess the mutual benefits of the GPS- and K-Band technique for gravity field 
determination. The sole use of the difference degree amplitudes (1) is not sufficient for a refined analysis.

The next series of solutions combine GPS and K-Band NEQs using the weight ratio of $\sigma_{p h}^{2} / \sigma_{k b d}^{2}=10^{8} \mathrm{~s}^{2}$. They may be considered as the normal or standard GRACE solutions. The results are expected to improve w.r.t. those obtained using only GPS or K-Band. Figure 7 shows that this is the case, but not by a large margin (when compared to the K-Band-only contribution). For reference, the corresponding solutions based only on K-Band are included (dash-dot lines, same color).

The pattern is rather similar for both types of solutions. The combined solution is slightly superior, in particular for the low degree terms.

The differences between the K-Band-only and the combined solution are shown in Fig. 8 for a 15 min spacing of the pulses. The differences show maximum (absolute) values for

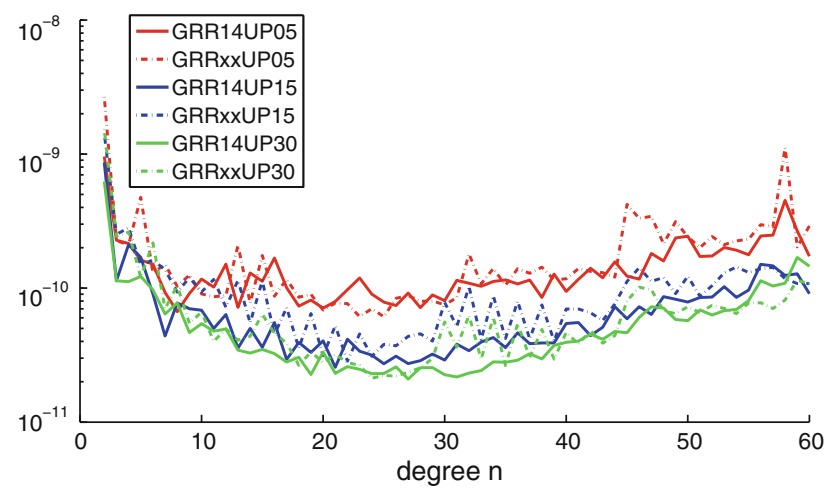

Fig. 7 Difference degree amplitudes w.r.t. AIUB-GRACE02S of the combined GPS- and K-Band (range-rate)-solution (weight ratio = $1 \times 10^{8}$ ); Corresponding K-Band-only solutions included for reference (dash-dot)

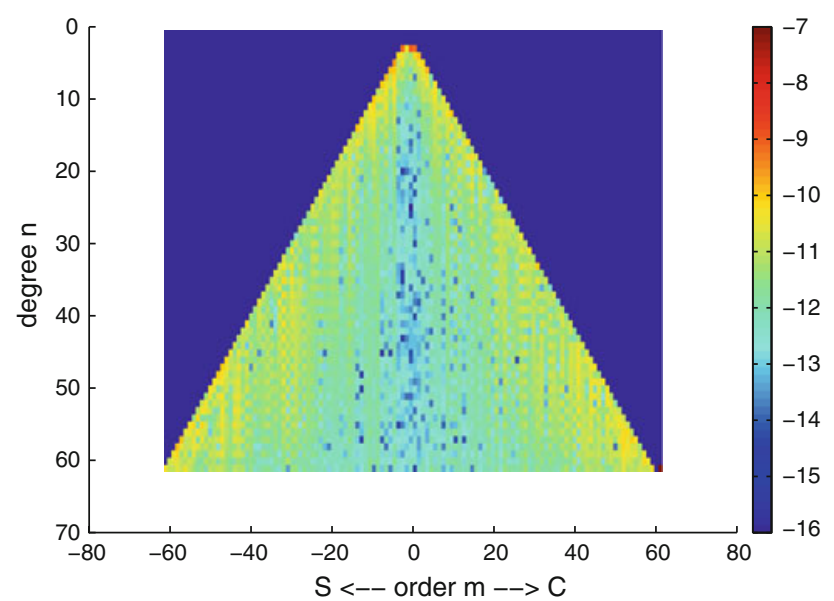

Fig. 8 Difference between spherical harmonic coefficients of combined and K-Band-only solutions (short-arcs of $15 \mathrm{~min}$ ) (weight ratio $=1 \times 10^{8}$ ) the (close to) sectorial terms, whereas the differences are on the RMS level or below for the (close to) zonal terms-except for the terms of very low degree.

Figure 8 shows that GPS has a significant impact on the gravity field determined from the data of the GRACE mission, provided the ratio $\sigma_{p h}^{2} / \sigma_{k b d}^{2}$ is defined according to Sect. 4. For $\sigma_{p h}^{2} / \sigma_{k b d}^{2} \rightarrow \infty$ the combined gravity field will asymptotically approach the K-Band-only case.

\section{Constraining pulses or accelerations in the CMA}

Figure 7 showed that the consistency of the 50-day solution with solution AIUB-GRACE02S increases with increasing spacing $\Delta t_{p}$ of the pulses, which points to an over-parametrization when reducing the pulse spacing to values below $10 \mathrm{~min}$. As mentioned it was necessary to weakly constrain the pulses for the 5 min solution in order to avoid true singularities. Whereas constraining was only applied to avoid singularities in Sect. 5, this option is used here to constrain the pulses as tightly as allowed by the "physical facts".

Figure 9 shows the difference degree amplitudes of two constrained solutions based on the $5 \mathrm{~min}$ pulse solution. In solution GRR14UP051610 (red curves) all pulses are constrained to $\sigma_{v_{l}}(\Delta t)=10^{-6} \mathrm{~m} / \mathrm{s}$ (RMS error a priori of the range-rate observations $\sigma_{0}=2 \times 10^{-7} \mathrm{~m} / \mathrm{s}$ ), in solution GRR14UP051612 (blue curves) the mean values of the pulses [see Beutler et al. 2010, Eq. (33)], are constrained to the same value of $\sigma_{v_{l}}(\Delta t)$, and half of the differences of the pulses are more tightly constrained to $10^{-8} \mathrm{~m} / \mathrm{s}$. Note that velocity changes of $10^{-6} \mathrm{~m} / \mathrm{s}$ with a spacing of $300 \mathrm{~s}$ are roughly equivalent to piecewise constant accelerations of $10^{-6} / 300 \approx 3.3 \times 10^{-9} \mathrm{~m} / \mathrm{s}^{2}$ with the same spacing [see Beutler et al. 2010, Eq. (17), a value which is clearly above the value recommended by Beutler et al. 2010, Eq. (19)]. The

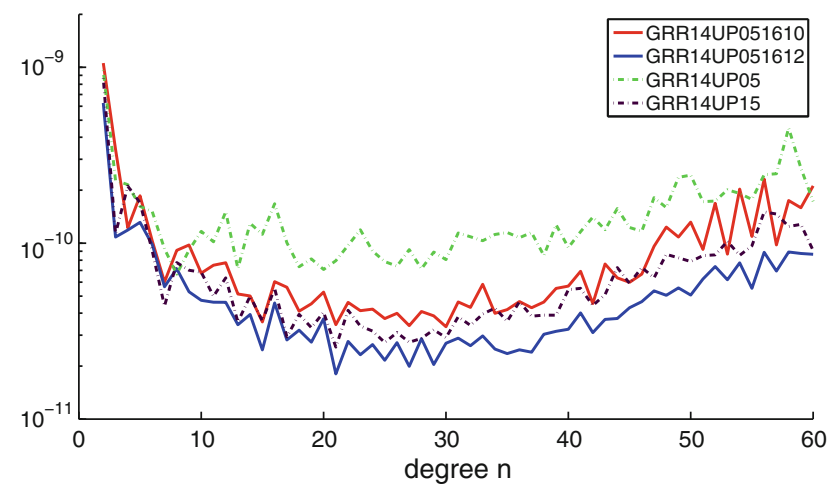

Fig. 9 Difference degree amplitudes of combined and constrained short-arc solutions (short-arcs of $5 \mathrm{~min}$ ) w.r.t. AIUB-GRACE02S. 5 and 15 min unconstrained solutions included for reference (dash-dot curves) 
large value allows it to absorb possible systematic effects in the kinematic positions emerging from GPS. The constraints applied to the pulse differences are two orders of magnitude smaller than the constraints applied to the mean values of pulses for solution GRR14UP051612, assuming that the errors to be absorbed by the pulses are highly correlated for GRACE-A and GRACE-B.

For reference the unconstrained (weakly constrained) solutions for the 5 and the 15 min short-arcs are included, as well (green and black curves). Constraining a parameter to zero with infinite weight is equivalent to leaving it out from the solution. This explains why the solutions GRR14UP051610 and GRR14UP051612 are closer in quality to the GRR14UP15 solution (i.e., to the unconstrained $15 \mathrm{~min}$ solution). Observe that the consistency with AIUB-GRACE02S is best for solution GRR14UP051612.

In analogy to Sect. 4 we should generate and discuss the solutions based on piecewise constant, constrained accelerations (as opposed to pulses). As the results are very close to those based on pulses-provided the constraints are adapted according to (Beutler et al., 2010, Eqs. (17)) — this discussion is skipped.

\section{Using accelerometer data in the CMA}

Figure 10 shows the accelerometer values for DOY 90/2007 (mean offsets removed, left: full day, right: 3-h detail view) in $\mathrm{m} / \mathrm{s}^{2}$ from top to bottom in the $R$-, $S$-, and $W$-directions for GRACE-A (red), GRACE-B (blue), and for the differences GRACE-A minus GRACE-B (green). The original measurements were transformed from the instrument frame to refer to the $R$-, $S$ - and $W$-directions. The GRACE-B data were moreover shifted by $\Delta t=+28 \mathrm{~s}$ before taking the differences $(\Delta t$ varies slowly from day to day). This time shift of the GRACE$B$ data makes the differences GRACE-A minus GRACE-B refer (roughly) to the same point in space. Assuming that the atmosphere and the insolation did not change much during $\Delta t=28 \mathrm{~s}$ and that the spacecrafts were not accelerated artificially during this time interval (e.g., by thruster firing), the accelerometers of the two satellites should measure almost the same signal and the difference of the series should be at least an order of magnitude smaller than the individual signals measured by the GRACE-A and -B accelerometers.

Figure 10 shows that this expectation is best met for $S$, not so well for $R$, and not at all for $W$ (all figures are drawn using the same scale). The difference signal in $S$ is generally well below the $10^{-9} \mathrm{~m} / \mathrm{s}^{2}$ level (after removal of a constant offset) and the small spikes usually may be attributed to one of the two satellites. In $R$, the signal common to both spacecrafts is clearly visible, but frequent spikes on the level of about $1-2 \times 10^{-8} \mathrm{~m} / \mathrm{s}^{2}$ (attributable to one of the two satellites and thus not reduced by forming the differences) do occur. In the $W$-direction the "true" difference signal is heavily affected by pulses frequently reaching values of $>5 \times 10^{-8} \mathrm{~m} / \mathrm{s}^{2}$. The characteristics of the spikes is visible particularly well in Fig. 10 (right). By consulting the thruster information file (THR1B*-files), described in Bettadpur (2007), one can associate virtually all of these spikes in $R, S$, and $W$ by (series of) thruster firings leaving their traces in the accelerometer data files. The crucial question is of course, whether these accelerations are real or not. Based on studies of many figures of type Fig. 10 the accelerometer measurements in the $W$-direction were excluded, because

- the pattern observed for DOY 90/2007 is typical for all other days of our data set;

- it cannot be excluded that the significantly larger sizes of the spikes in $W$ are due to the reduced measurement accuracy in this direction (Touboul et al. 1999);

- the $W$-measurements only have a small impact on the $\mathrm{K}$-Band measurements.

Tests made using and not using the $W$-accelerations did not reveal significant quality differences.

The differences of the along-track accelerations of GRACE-A and GRACE-B govern the time development (due to the non-gravitational forces) of the distance between the satellites. The differential along-track signal in Fig. 10 (middle row, green) shows the differential signal (essentially) at the same location. For describing the inter-satellite distance the plain difference without time shift has to be used. The corresponding figure was not included in the interest of shortening the article.

Our solutions use accelerometer data in a naive sense, by just interpreting the data in the accelerometer files as values of a given empirical function in the numerical integration process. Parameters which may be considered as accelerometer-specific in the CMA are: offsets and once-per-revolution parameters for the $R$-direction, a polynomial of degree $q=3$ and once-per-revolution parameters for the $S$-direction. The pulses in $R$ and $S$ may be viewed as accelerometer-specific, as well. Constant, once-per-revolution parameters, and pulses are also set up for the $W$-direction. As the accelerometer measurements in the $W$-direction are not used, the mentioned parameters define the empirical accelerations in the $W$-direction. In solutions not making use of accelerometer data the same parameters in the $R$ - and $S$-directions are set up as in the solutions using accelerometer data, but the interpretation is different: they are now the parameters of the empirical acceleration model in $R$ and $S$.

For gravity field estimation without and with accelerometers the pattern set in Sect. 5 is followed: first, the GPS-only solutions without using accelerometer data are generated and 

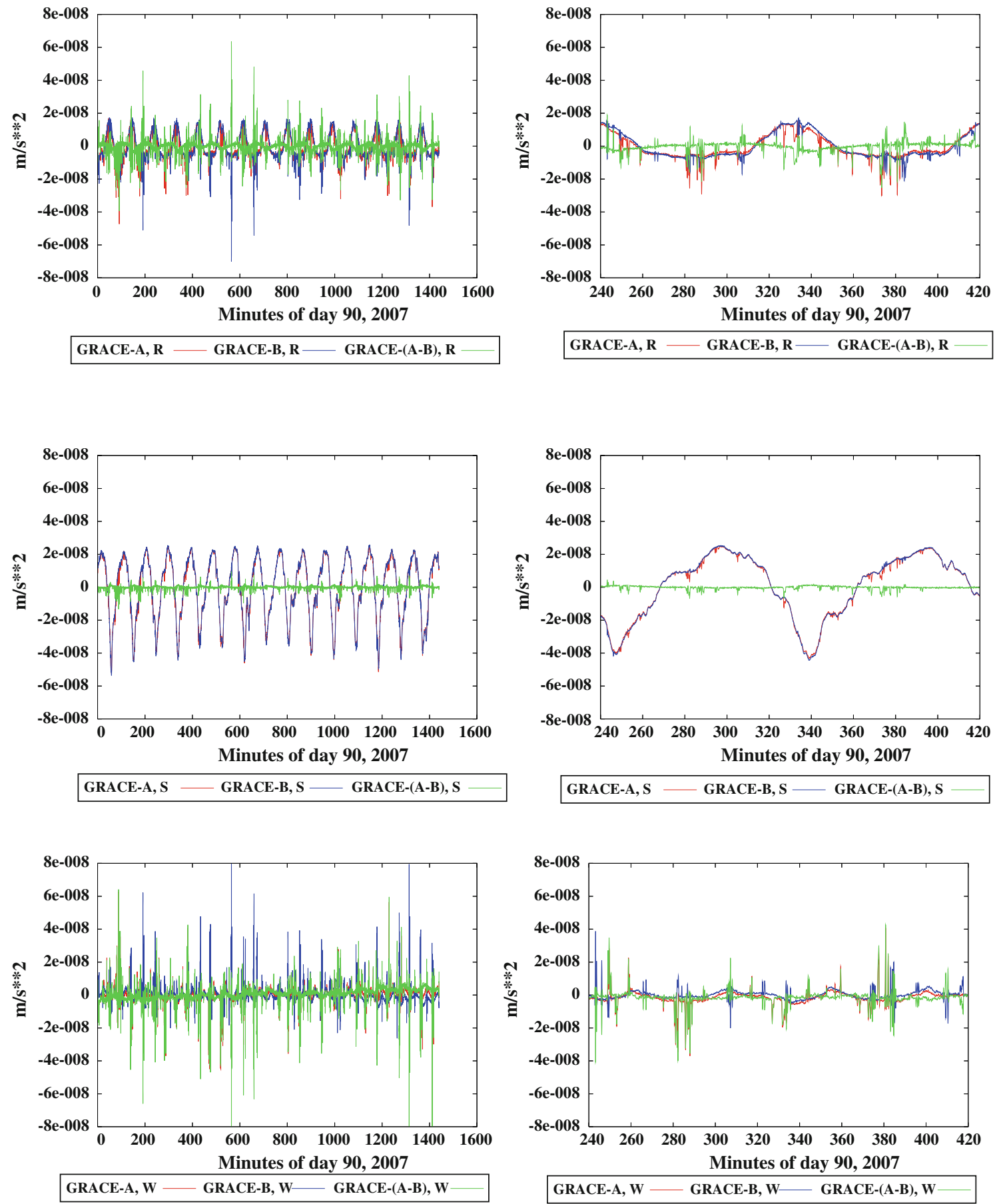

Fig. 10 Accelerometer measurements in $R$-, $S$-, and $W$-directions for GRACE-A, GRACE-B and the difference GRACE-A -B, time shifted to refer the measurements to "the same" point in space. Left: full DOY 90/2007, right: $4^{h}-7^{h}$, same day

compared to the corresponding solutions making use of the accelerometer data. Then, the same analysis is performed with the combined GPS- and K-Band solutions. Apart from not using the accelerometer data the background models and the parametrization for the new solutions are identical with those documented in Sect. 5. We thus expect to isolate the net impact of the accelerometer data.
Let us start with the GPS-only results: there is no visible effect in the difference degree amplitudes, when comparing the solutions with and without accelerometer data with an analogue to Fig. 3. Small differences do, however, exist. Figure 11 visualizes these differences (absolute values, logarithmic scale) for the 15 min solutions using a color code. We conclude that the accelerometer data have very little impact 


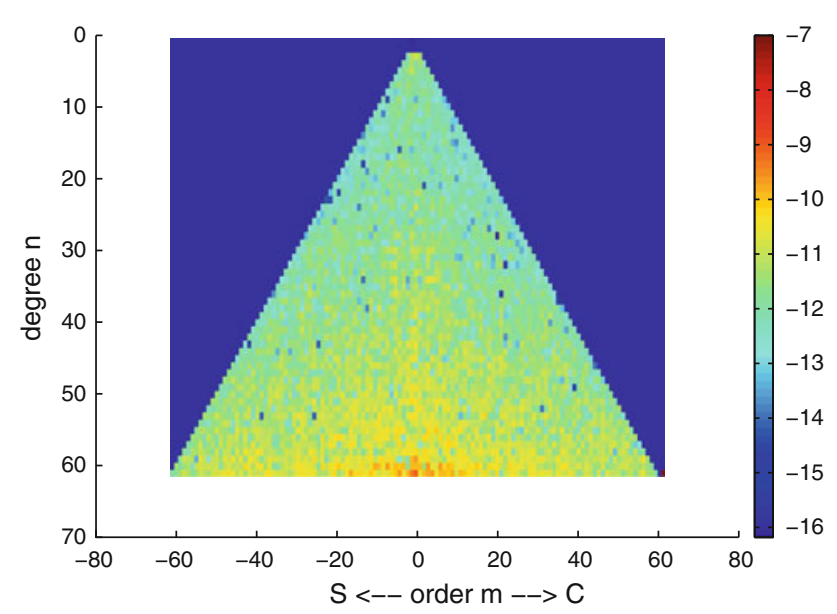

Fig. 11 Effect of the accelerometer data on spherical harmonics coefficients for the $15 \mathrm{~min}$ GPS-only solutions (absolute values, logarithmic scale)

on the quality of the gravity field using only the GPS measurements. This result confirms the findings of Ditmar et al. (2007) and Prange et al. (2009).

Figure 12 (left), showing the difference degree amplitudes w.r.t. solution AIUB-GRACE02S for the three short-arc solutions with a spacing of pulses of $\Delta t=5,15,30 \mathrm{~min}$ neglecting the accelerometer data (solid lines) and the corresponding solutions making use of the accelerometer data (dash-dot lines), underlines that the accelerometer measurements have a significant impact on gravity field determination when using in addition K-Band range-rates, but that solutions of good quality may also be generated without using these measurements. This result confirms the finding of Jäggi et al. (2010). The quality gain is more pronounced for the higher degrees than for the lower degrees, because the accelerometer-specific parameters successfully remove the low-, but not the high-frequency systematics. The impact of the acceler-

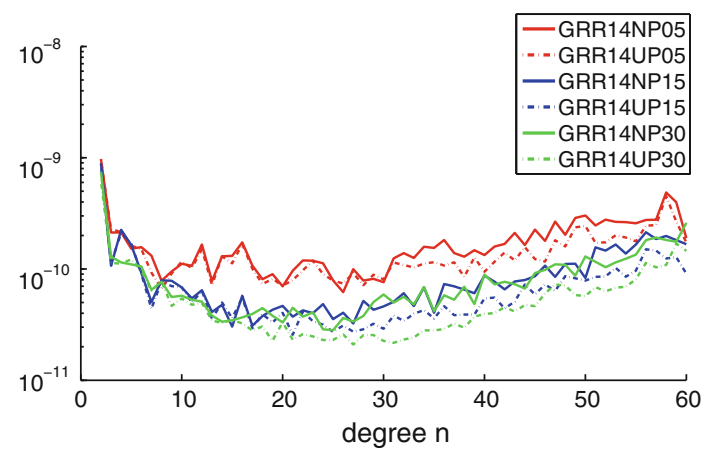

Fig. 12 Combined GPS- and K-Band solutions (weight ratio $1 \times$ $10^{8} \mathrm{~s}^{2}$ ). Left: Difference degree amplitudes w.r.t. AIUB-GRACE02S (solid lines solutions without accelerometers, dash-dot lines solutions ometer data becomes more and more prominent with increasing spacing $\Delta t_{p}$ of the pulses. For the $30 \mathrm{~min}$-arcs the level of the degree difference amplitudes w.r.t. AIUB-GRACE02S is reduced by about $0.15-0.30$ in the logarithmic scale, corresponding to a factor of about 1.4-2.0 in the numeric scale. Figure 12 (right) visualizes the differences of the individual terms estimated with and without the use of the accelerometer data. The differences are very small for the low order terms, they are rather pronounced for the (close to) sectorial terms and the low-degree terms.

\section{Analyzing K-Band ranges, range-rates, and range-differences}

All solutions involving K-Band were till now based on the range-rate observable without modeling the mathematical correlations between the observations. Alternative solutions are studied in this section and they are put in relation to the range-rate solution. Table 3 lists the processing options offered by the CMA. Let us point out that all experiments performed here are based on the assumption of white noise for the original Level-1A range data. Some results involving difference degree amplitudes might be slightly different (worse) when dropping this assumption. The results involving error degree amplitudes are not affected.

Range (options $R$ and $R C$ ), range-differences (options $R D$ and $D D$ ), or range-rate (options $R R$ and $R R C$ ) may be used as the basic K-Band observables. Options $R, R D, D D$, and $R R$ disregard the mathematical correlations introduced by the filtering process to generate Level $1 \mathrm{~b}$ from Level $1 \mathrm{a}$ data [Beutler et al. 2010, Sect. 3.3], options $R R C$ and $R C$ take them into account. The generic formulas for filtering are represented by (Beutler et al., 2010, Eqs. (6)). The resulting weight matrices for range and range-rate Level $1 \mathrm{~b}$ data are given by (Beutler et al., 2010, Eqs. (8) and (9)), respectively.

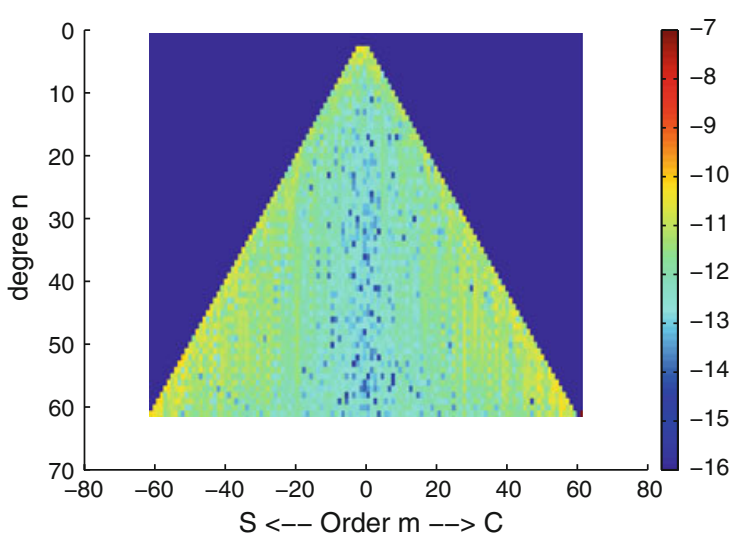

with accelerometers); right: difference of estimated spherical harmonics coefficients with and without accelerometer data for the $15 \mathrm{~min}$ solution 
Table 3 CMA processing options for K-Band Analysis

\begin{tabular}{lll}
\hline Option & Correlations & K-band observable \\
\hline RR & No & Range-rates \\
R & No & Ranges \\
RD & No & Range differences \\
DD & No & Range-double-differences \\
RRC & Yes & Range-rates \\
RC & Yes & Ranges \\
\hline
\end{tabular}

The explicit GRACE filter formulas are given in Thomas (1999).

Formulas $(8,9)$ in Beutler et al. (2010) suggest that a correct processing of a batch of Level $1 \mathrm{~b}$ K-Band data (in our case a batch corresponds to one day) implies a fully populated weight matrix $\mathbf{P}$ for range and $\mathbf{P}^{\prime}$ for range-rate, respectively, where the dimension of the weight matrices equals the number of observations. This dimension is $d=86,400 / 5=$ 17,280 when analyzing a full day of $5 \mathrm{~s}$ data. It is in principle trivial to implement a procedure to handle correlations correctly. Such procedures will, however, become rather inefficient when data spans of the order of one day or more are dealt with. To the best of our knowledge, nobody made the attempt to implement correct correlations over intervals of this length. We do not have the intention to do that eitheralso based on the results of this section.

It is, however, easily possible to subdivide the entire time interval into shorter subintervals of, let us say, $30 \mathrm{~min}$ to $2 \mathrm{~h}$, and to use the correct correlation matrices $\mathbf{P}$ or $\mathbf{P}^{\prime}$ within these subintervals. This subdivision may, but need not, be synchronized with the stochastic epochs $t_{p}$. This procedure has to be understood in the CMA under options $R C$ and $R R C$ for ranges and range-rates, respectively, where the length of the subintervals is set by the user. In order to get a first idea of the relevance of correlation modeling the matrices $\mathbf{P}$ and $\mathbf{P}^{\prime}$ are visualized for a time interval of $30 \mathrm{~min}$ and a data spacing of $\Delta t=5 \mathrm{~s}$.
Figure 13 (left) shows that the weight matrix $\mathbf{P}$ for range is "almost diagonal". It is even closely related to $\mathbf{P} \approx s \times \mathbf{U}$, where $s$ is a positive scaling factor and $\mathbf{U}$ is the unit matrix, where the dimension equals the number of K-Band observations in the subinterval. Using either matrix $\mathbf{P}$ or its approximation (scaled unit matrix) therefore cannot have too much of an impact on the results. Options $R$ and $R C$ therefore are expected to give similar results. Evidence for this statement will be provided later in this section.

Figure 13 (right) shows that the weight matrix $\mathbf{P}^{\prime}$ for range-rate is far from diagonal. Therefore, one concludes at first sight that correlations must be taken into account when analyzing this observable. One may ask the question, however, whether option $R R C$ is needed at all, when options $R$ and $R C$ are available: Apart from first and higher order terms in $\Delta t$ range-rate equals range-difference between subsequent ranges divided by the time difference $\Delta t$ separating the ranges

$\dot{\rho}_{i}=\frac{\rho_{i+1}-\rho_{i}}{\Delta t}+O(\Delta t)$,

where $\Delta t=5 \mathrm{~s}$ for the GRACE Level $1 \mathrm{~b}$ data. To the same order in $\Delta t$ the second time derivative of range may be approximated as

$\ddot{\rho}_{i}=\frac{\rho_{i+2}-2 \rho_{i+1}+\rho_{i}}{\Delta t^{2}}+O(\Delta t)$.

Equation (5) simply says that analyzing range-double-differences and range-accelerations (both without taking correlations into account) must give almost identical resultsassuming that the factor $\Delta t^{2}$ is taken into account in the weight matrix when combining the GPS- and K-Band-specific NEQs. With option $D D$ it is thus possible to obtain (almost) identical results as those, which would emerge in an analysis of range-accelerations. The CMA is thus "in principle" capable of generating orbits and gravity fields based on ranges, range-rates and range-accelerations using the options $R, R D, D D$. Gravity fields based on the $R, R D$, and $D D$
Fig. 13 Weight matrices for a time interval of $30 \mathrm{~min}$ with $\Delta t=5 \mathrm{~s}$ data spacing (left: $\mathrm{K}$-Band ranges, right: K-Band range-rates)
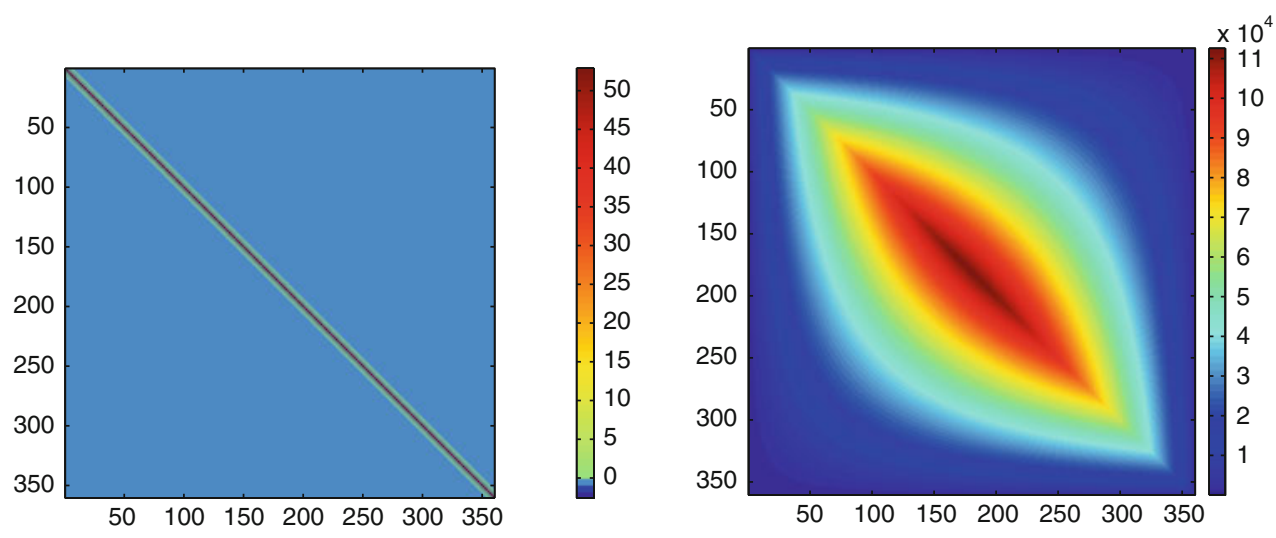
options will be provided at the appropriate place in this section. Equation (4) has the following messages:

- The processing options $R R$ and $R D$ are closely related. One obtains, as a matter of fact, almost identical gravity fields (evidence provided below) when using weight factors for the GPS/K-Band combination of NEQs according to Eq. (4) when analyzing range-rate or range-differences (both without modeling correlations), respectively.

- The simultaneous use of ranges and range differences adds no independent information to an analysis based on ranges, because the latter observables are linear combinations of the former - if the weights of the range-difference observations are calculated by applying the law of error propagation to the linear combination of ranges on the right-hand side of Eq. (4).

- The situation is different, if the weights are set in a different way, e.g., by assigning a much higher weight to range-differences as compared to ranges (than that emerging from Eq. (4)). The CMA allows it to combine range, range-rate solutions, range-accelerations on the NEQlevel with user-defined weights. This option was, however, not studied in this work.

- Equation (4) helps to understand Fig. 13 (right): assuming that the Level $1 \mathrm{~b}$ ranges are independent (not too bad an assumption by virtue of Fig. 13 (left)) with variance $\sigma^{2}=1$, the relation between the range differences and the ranges reads as

$$
\Delta \rho=\left(\begin{array}{cccccc}
-1 & 1 & 0 & \ldots & 0 & 0 \\
0 & -1 & 1 & \ldots & 0 & 0 \\
\ldots & \ldots & \ldots & \ldots & \ldots & \ldots \\
\ldots & \ldots & \ldots & \ldots & \ldots & \ldots \\
0 & 0 & \ldots & -1 & 1 & 0 \\
0 & 0 & \ldots & 0 & -1 & 1
\end{array}\right) \rho \doteq \mathbf{D} \rho
$$

Under the assumptions mentioned the covariance matrix of the range-differences is $\operatorname{cov}(\Delta \rho)=\mathbf{D D}^{T}$, a block diagonal symmetric matrix with only the diagonal and the adjacent elements to the diagonal different from zero. Its inverse $\left(\mathbf{D D}^{T}\right)^{-1}$, however, has almost exactly the shape of Fig. 13 (right)! The shape of matrix $\mathbf{P}^{\prime}$ is thus mainly determined by forming the time differences of the Level $1 \mathrm{~b}$ ranges and does not have much to do with the filtering process to generate the Level $1 \mathrm{~b}$ range-rates from the Level 1a ranges.

Based on these facts options $R$ and $R R C$ are expected to provide almost identical results:

1. Biased ranges without correlations (option $R$ ) and rangedifferences with weight matrix $\left(\mathbf{D D}^{T}\right)^{-1}$ (not available as a separate option in the CMA) will inevitably lead to identical results.

2. Because of Eq. (4) the analysis based on range-rate using the correlation matrix $\Delta t^{2}\left(\mathbf{D D}^{T}\right)^{-1}$ will generate, apart from terms of higher than second order in $\Delta t$, identical results as that based on range-differences with matrix $\left(\mathbf{D D}^{T}\right)^{-1}$ as weight matrix.

3. As the correct weight matrix for range-rates and its approximation $\Delta t^{2}\left(\mathbf{D D}^{T}\right)^{-1}$ are very similar, we are eventually allowed to conclude that options $R$ and $R R C$ must give almost the same results, as well (evidence provided below).

As we have already dealt with option $R R$, we can in principle only hope for the analysis of ranges (options $R$ and $R C$ ) to achieve better (more carefully: other) results than with an analysis based on option $R R$. As options $R, R D, D D, R C$, and $R R C$ are of theoretical interest, as well, we include one gravity field solution based on range-differences, one on range-double-differences, one based on ranges using correct correlations, one based on range-rates using correct correlations, and a few solutions based on ranges without modeling correlations.

The gravity fields are determined, to the extent possible, in a way consistent with that of Sect. 5 based on option $R R$ : For this purpose the statistical information (weight ratio of GPS and K-Band and a priori RMS of the weight unit) from option $R R$ is transformed into information suitable for processing range-differences, range-double-differences, ranges, and range-rates with correlations. Options $R$ and $R D$, both neglecting correlations, will be used to analyze range and range-difference data, respectively. In Fig. 1 we found

$$
\left(\frac{\sigma_{k b d, r r}}{\sigma_{p h}}\right)^{2}=\left(\frac{0.2 \times 10^{-6}}{2 \times 10^{-3}}\right)^{2} \approx 1.0 \times 10^{-8} \mathrm{~s}^{-2},
$$

Using Eq. (4) we translate this relation into the following ratio for range-differences spaced by $\Delta t=5 \mathrm{~s}$ :

$$
\left(\frac{\sigma_{k b d, r d}}{\sigma_{p h}}\right)^{2}=\left(\frac{5 \times 0.2 \times 10^{-6}}{2 \times 10^{-3}}\right)^{2} \approx 2.5 \times 10^{-7} .
$$

Assuming that the ranges needed to form the range differences are independent-in view of Fig. 13 (left) this is not too far from the truth-we are left with

$$
\left(\frac{\sigma_{k b d, r}}{\sigma_{p h}}\right)^{2}=\left(\frac{\sqrt{2} / 2 \times 10^{-6}}{2 \times 10^{-3}}\right)^{2} \approx 1.2 \times 10^{-7}
$$

for the individual Level $1 \mathrm{~b}$ ranges. We can now easily add the consistent weight ratio when analyzing weight 
double-differences (the factor $\sqrt{6}$ follows from Eq. (5)):

$$
\left(\frac{\sigma_{k b d, r d d}}{\sigma_{p h}}\right)^{2}=\left(\frac{0.7 \times 10^{-6} \times \sqrt{6}}{2 \times 10^{-3}}\right)^{2} \approx 7.2 \times 10^{-7} \text {. }
$$

When using options $R C$ and $R R C$ the $\mathrm{K}$-Band RMS refers to the Level 1a range. In order to get comparable results with the other solutions one has to modify the ratio for combining the K-Band range and GPS NEQs according to

$$
\left(\frac{\sigma_{k b d, r, 1 a}}{\sigma_{p h}}\right)^{2}=\left(\frac{7.25 \times 0.7 \times 10^{-6}}{2 \times 10^{-3}}\right)^{2} \approx 6.4 \times 10^{-6}
$$

The factor of 7.25 of noise reduction from Level 1a to Level $1 \mathrm{~b}$ ranges and range-rates may be extracted from the concrete filter formulas in Thomas (1999). An order of magnitude calculation gives approximately the same answer: the GRACE filter for generating Level $1 \mathrm{~b}$ ranges may be very crudely approximated by a moving average over a $5 \mathrm{~s}$ interval containing 50 observations. The RMS of the mean value would thus be $\sqrt{50} \approx 7.1$.

The RMS values a priori for the individual observables are:

$$
\begin{aligned}
& \sigma_{p h}=2 \mathrm{~mm} \\
& \sigma_{k b d, r, 1 a}=5 \mu \mathrm{m} \\
& \sigma_{k b d, r}=0.7 \mu \mathrm{m} \\
& \sigma_{k b d, r d}=1.0 \mu \mathrm{m} \\
& \sigma_{k b d, r d d}=1.7 \mu \mathrm{m} \\
& \sigma_{k b d, r r}=0.2 \mu \mathrm{m} / \mathrm{s}
\end{aligned}
$$

Figure 14 shows the difference degree amplitudes of the range-difference solution GRD54UP30 w.r.t. the range-rate solution GRR14UP30. The former solution was produced

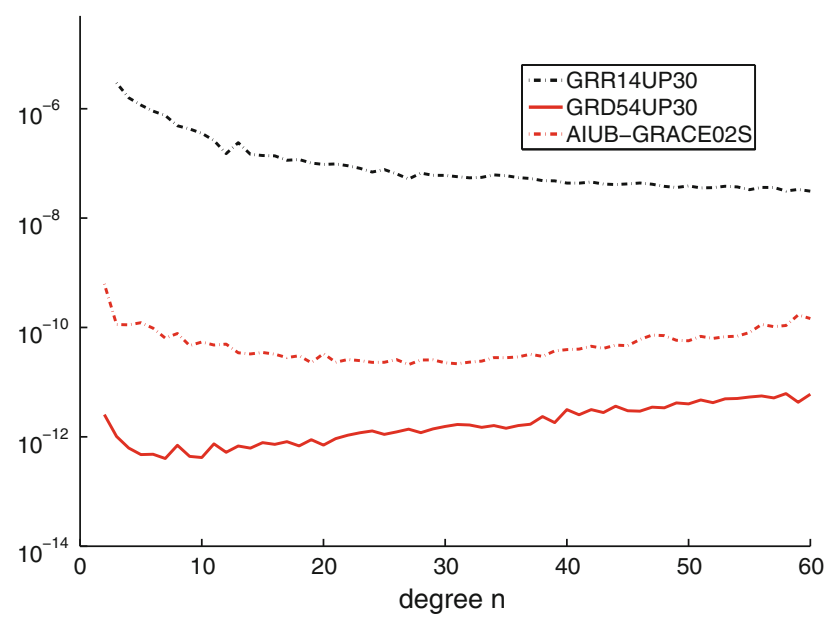

Fig. 14 Difference degree amplitudes of solution GRD54UP30 w.r.t. solution GRR14UP30, solution AIUB-GRACE02S included for reference with option $R D$, the latter with $R R$. Both solutions are based on pulses with a spacing of $30 \mathrm{~min}$ and ignore correlations. The differences are well below the difference degree amplitudes between solutions AIUB-GRACE02S and GRR14 UP30 (by a factor of 10 or more), which represent the consistency of solutions GRR14UP30 and/or GRD54UP30 with the biennial solution AIUB-GRACE02S. The message of this figure is simple: Options $R R$ and $R D$ generate close to identical results when using Eqs. (8) and (7), respectively, for the mutual weighting of the K-Band and GPS-NEQ contributions. Consequently, it would not have been necessary to include range-rate (neither range-acceleration) in the GRACE Level $1 \mathrm{~b}$ data. This result is of interest, as well, because the analysis of range-differences is a way to circumvent the numerical differentiation process for generating the range-rate observable from ranges (see Beutler et al. 2010, Eq. (6)).

Figure 15 shows that the correct modeling of correlations or the assumption of independently measured Level $1 \mathrm{~b}$ ranges, respectively, does not matter from the standpoint of the practitioner: The difference degree amplitudes between the two solutions in Fig. 15, generated with options $R$ and $R C$, are well below the degree difference amplitudes of the individual solutions (with or without correct correlation modeling) w.r.t. the biennial solution AIUB-GRACE02S.

Figure 16 compares the range solution GRG33UP30 (reference) with correct correlations with the range-rate solution GRR33UP30 with correct correlations. The difference degree amplitudes of the biennial solution AIUB-GRACE 02S with solution GRG33UP30 are included for reference. The two solutions with correct correlations generated with options $R C$ and $R R C$, respectively, may be considered as equivalent from the point of view of the practitioner. As we already showed the equivalence of options $R$ and $R C$ we may

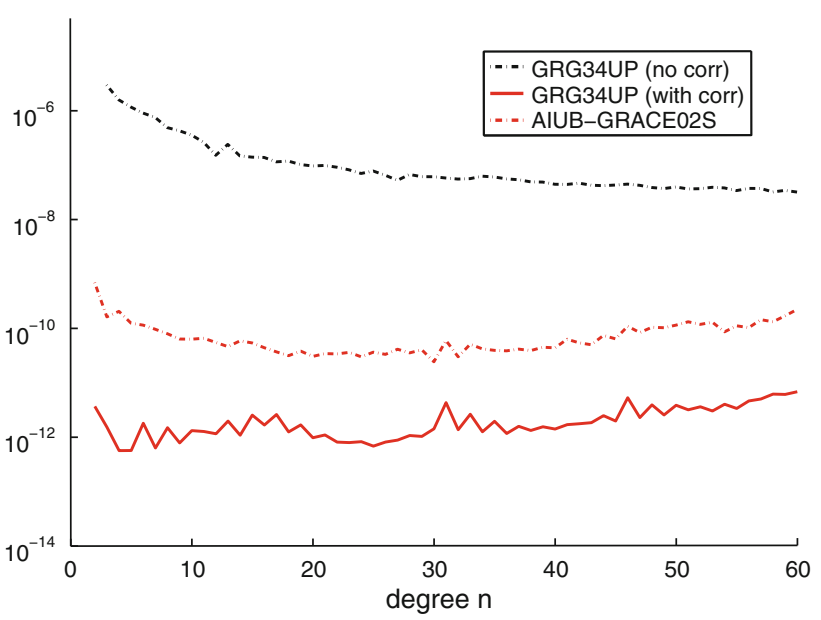

Fig. 15 Difference degree amplitudes of solution GRG33UP30 (with correlations) w.r.t. solution GRG34UP30 (without correlations), solution AIUB-GRACE02S included for reference 


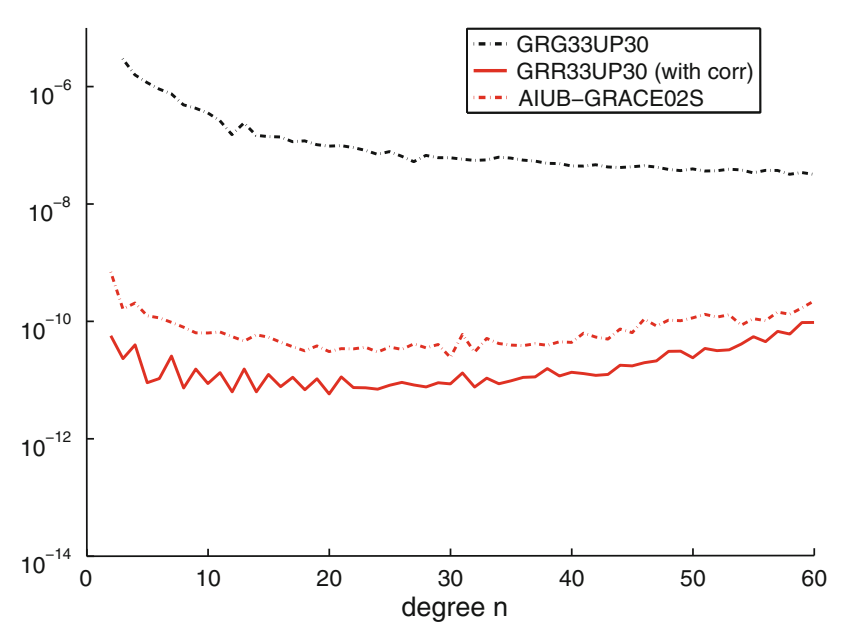

Fig. 16 Difference degree amplitudes of solution GRR33UP30 (range-rate with correlations) w.r.t. solution GRG33UP30 (range with correlations), solution AIUB-GRACE02S included for reference

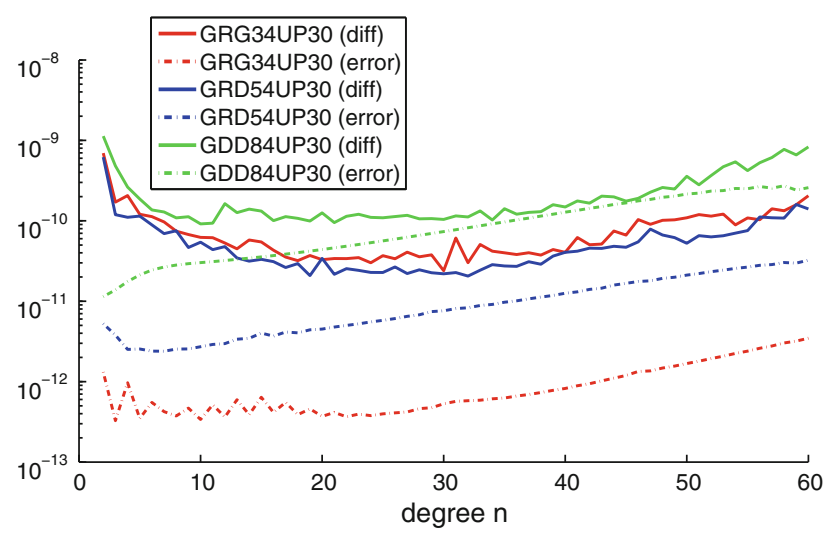

Fig. 17 Difference degree amplitudes of solutions GRG34UP30, GRD54UP30, GDD84UP30 w.r.t. solution AIUB-GRACE02S; Error degree amplitudes of the three range solutions included (a priori RMS values used to define weights)

also conclude that options $R, R C$, and $R R C$ are equivalentprovided the parametrization is the same and the mutual weighting of the K-Band and GPS contributions follows the rules of this section. The result is based on the assumption that the Level $1 \mathrm{~b}$ ranges and range-rates are derived from one and the same set of Level 1a ranges. If range rates were measured independently (and not generated by taking the derivatives of the ranges), the result would be different.

Figure 17 shows the consistency of the solutions produced with options $R, R D, D D$ with the solution AIUBGRACE02S. Having shown that the solutions $R D$ and $R R$ are equivalent (see Eq. (4) and Fig. 14) and that the analysis of range acceleration and of range-double-differences (see Eq. (5)) are equivalent, Fig. 17 also illustrates the mutual values of the range, range-rate, and range-acceleration observ- ables. The solid lines illustrate what was achieved in relation to the solution AIUB-GRACE02S (a) with the range solution $(R$, red), (b) the range-difference solution $(R D$, blue), and (c) the range-double-difference solution ( $D D$, green). The three alternative K-Band contributions were combined individually with the GPS contribution using the weight ratios in Eq. (9) for range, in Eq. (8) for range-difference, and in Eq. (10) for range-double-difference. Except for the range biases in the range solution $R$ the parametrization was the same in the three solutions (unconstrained pulses with a spacing of $30 \mathrm{~min}$, correlations of the K-Band observables ignored). The range-difference solution ( $R D$, blue, solid line) is clearly the best choice for the particular parametrization, followed by $R$ (red, solid) in second and $D D$ (green, solid) in the third place. The range solution $R$ and the range-double-difference solution $D D$ are of lesser quality (consistency with the biennial solution), for different reasons, however. This becomes clear by comparing the error degree-amplitudes (dash-dot lines) of the three solutions, generated with the RMS errors a priori in Eq. (12), with the corresponding difference-degree amplitudes (solid lines, same color). The two green curves are close to each other, implying that the range-double-difference observable is not capable of providing more information, but also that the white noise dominates systematic errors. The $R$ solution (red) promises excellent results (dash-dot), provided the model adequately represents the ranges on the $0.7 \mu \mathrm{m}$ level. This is obviously not the case-by almost two orders of magnitude! The $R D$ solution is a good compromise from the point of view of the practitioner: the theoretical expectations (blue, dash-dot) and the achieved accuracies (blue, solid) are not too far apart. The actual differences between the three solutions are relatively small for low degrees, because the GPS-contribution, which has a large impact in this domain, is the same in the three solutions.

\section{Analysis of the K-band residuals}

Let us further explore the characteristics of two solutions performed in the previous section, which were based on option $R$. Figure 18 shows the range residuals without (red) and with (blue) using accelerometer data of an orbit determination experiment for DOY 62/2007 based on pulses spaced by $30 \mathrm{~min}$. Whereas the reduction of the range-rate residuals was of the order of $10 \%$ when using the accelerometer data in the case of range-rates (not shown), the corresponding gain is more than a factor of $2(>100 \%)$ in the case of ranges. The range observable obviously is much more susceptible to acceleration-induced model deficiencies than the rangerate observable. This goes hand in hand with the "promised" higher accuracy when using the ranges under the assumption that there are no systematics on or above the level of the 


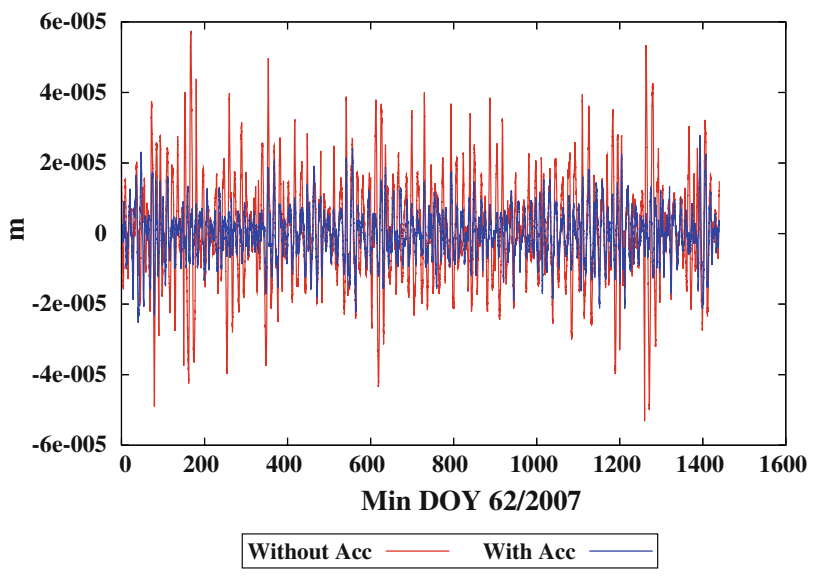

Fig. 18 K-Band range residuals, DOY 62/2007. Gravity fild determination without (red) and with (blue) accelerometer data

accuracy of the range measurements (see Fig. 17). Systematic effects (periodic excursions with periods of few minutes) are dominating the range residuals in Fig. 18. This was not the case when using option $R R$ (see Fig. 2). This difference explains, as well, why the analysis of range (be it for orbit or gravity field determination) is less successful than the analysis of range-rate. Despite the fact that a covariance analysis tells that the range observable should lead to much better results than the range-rate observable, the results are slightly inferior, because range is much more prone to (force) model deficiencies. This insight can be underpinned with the following thought experiment. Let us assume that there is a (non-modeled) effect

$\Delta \ddot{\rho}=\xi \cos \frac{2 \pi}{P} t$

of period $P$ and amplitude $\xi$ in the inter-satellite accelerations. Such a periodic signal approximately invokes a signal of the following kind in range-rate and in range, respectively,

$$
\begin{aligned}
& |\Delta \dot{\rho}|=\frac{P \xi}{2 \pi} \sin \frac{2 \pi}{P} t \\
& |\Delta \rho|=\frac{P^{2} \xi}{(2 \pi)^{2}} \cos \frac{2 \pi}{P} t
\end{aligned}
$$

implying that the amplitudes in the observables, as compared to the amplitude in the acceleration, are multiplied by a factor of $P /(2 \pi)$ (changing the units of the amplitude from $\mathrm{m} / \mathrm{s}$ to $\mathrm{m} / \mathrm{s}$ ) for range-rate and by the square of this value for range (changing the units of the amplitude from $\mathrm{m} / \mathrm{s}^{2}$ to $\mathrm{m}$ ). A non-modeled acceleration with amplitude $\xi=1 \times$ $10^{-9} \mathrm{~m} / \mathrm{s}^{2}$ and period $P=600 \mathrm{~s}$ thus translates into a nonmodeled effect in the range-rate residuals of $9.5 \times 10^{-8} \mathrm{~m} / \mathrm{s}$, and into a non-modeled effect of $9.1 \times 10^{-6} \mathrm{~m}$ in the range residuals. Whereas the resulting effect in range-rate is well hidden in the noise in the case of the GRACE range-rate observable, the effect is almost a factor 10 above the claimed accuracy level of the GRACE range observable. This short excursion explains why the prominent periods in the range (but also the range-rate) residuals are those of few minutes in our analysis. Non-modeled effects of longer periods are absorbed by the pseudo-stochastic (and/or dynamic empirical) parameters, whereas shorter period effects can only be absorbed by the gravity-field parameters-causing a significant deterioration of the gravity field.

Figure 19 shows that these order-of-magnitude results are confirmed by the spectra of the residuals. The amplitudes of the terms with periods around $P=10 \mathrm{~min}$ show the behavior expected according to Eqs. (13, 14). Figure 19 (left) shows the amplitude spectrum of the range-rate, Fig. 19 (right) that of the range residuals of days 50-99. One can see spectral lines with growing amplitudes for periods $0<P<15 \mathrm{~min}$. If the spectral lines were caused by non-modeled accelerations of the same amplitudes but with different periods one would expect, according to our argumentation, quadratically growing amplitudes in the ranges, linearly growing amplitudes in range-rate as a function of the period $P$. Figure 19 are not in conflict with this statement for periods up to about 15 min. A double-logarithmic version of Fig. 19 would show

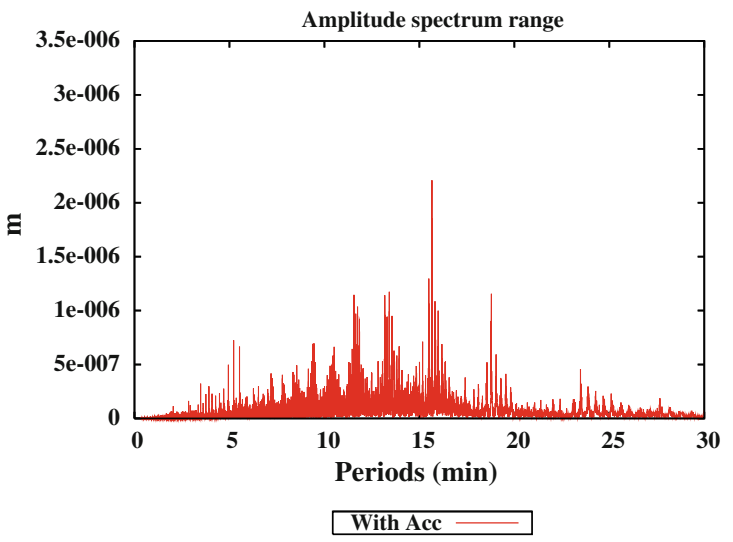

Fig. 19 Spectra of 50 day (DOY 50-99/2007) of range-rate and range residuals using the accelerometer data. Left: range-rate, right: range 


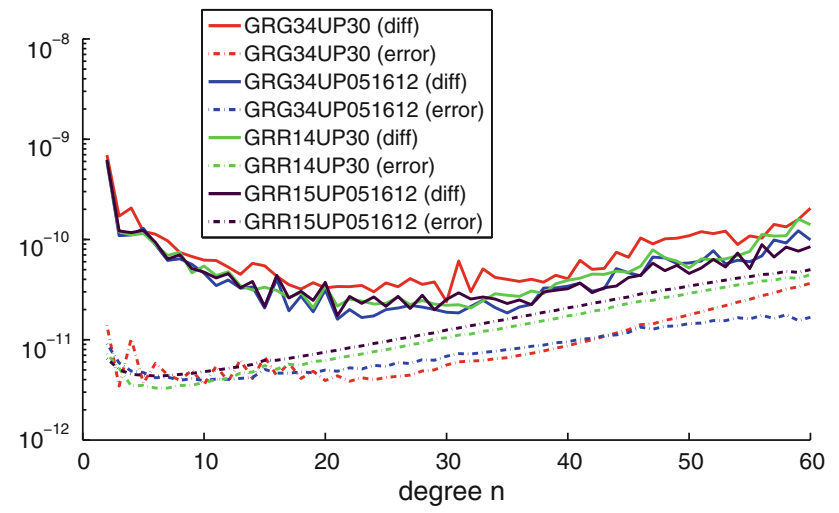

Fig. 20 Difference degree amplitudes (solid) and error degree amplitudes (dash-dot) of constrained range (blue) and range-rate solutions (black, pulse spacing of $5 \mathrm{~min}$ ) w.r.t. AIUB-GRACE02S. Red: short-arc 30 min range solution; Green: 30 min short-arc range-rate solution

that the growth of the amplitudes in the mentioned domain approximately meets the expectation (14). The attenuation of the amplitudes for periods $15 \mathrm{~min}$ is caused by the absorption of these periodic effects by the pseudo-stochastic parameters (and unfortunately the gravity field parameters).

\section{A range solution of good quality}

In this section we want to generate a gravity field solution based on ranges (option $R$ in Table 3 ), which is comparable in quality with the best range-rate solutions. The residuals of the 30 min range solution and the spectrum of the residuals associated with it (Fig. 19 (right)) indicate how this can be done: One has to absorb the spectral lines with periods $P<15 \mathrm{~min}$ by pulses using a spacing $\Delta t_{p} \ll 15$ min-without losing too much power for gravity field determination. This is possible by imposing constraints on the pulses spaced by, let us say, $5 \mathrm{~min}$.

This procedure was applied to the range-rate solutions (see Sect. 6, Fig. 9), but only with limited success: The constrained range-rate solution based on $5 \mathrm{~min}$ pulses was only slightly better than the solutions based on unconstrained pulses spaced by $15 \mathrm{~min}$.

The constrained range solution GRG34UP051612 (blue), to be compared to the constrained range-rate solution GRR15 UP051612 (black), is provided in Fig. 20. The difference degree amplitudes, represented by the solid lines, show that the range solution based on a 5 min spacing of constrained pulses (blue) is clearly better than the range solution with unconstrained $30 \mathrm{~min}$ pulses (red). The blue curve in turn is comparable in quality to the two range-rate solutions (green and black).

The error degree amplitudes in Fig. 20 (dash-dot lines) are based on the a posteriori RMS errors. The constrained range solution (blue) is comparable to the unconstrained $30 \mathrm{~min}$ solutions (red) for the low degrees, whereas the solution with constrained $5 \mathrm{~min}$ pulses promises to be slightly better for the higher degrees. This degree-dependent behavior is not confirmed by the error degree amplitudes (blue and red solid lines). When replacing the a posteriori by the a priori errors, the dash-dot red curve would be reduced by a factor of about 10 , whereas the dash-dot blue curve would be reduced "only" by a factor of about $10^{0.3} \approx 2$. The impact of the systematic errors on the constrained 5 min solution as compared to the unconstrained $30 \mathrm{~min}$ solution is thus considerably reduced, but at the price of losing resolution power for gravity field determination. By further reducing the spacing of the pulses the range residuals will eventually be free from systematic errors, but the determined gravity field will not improve substantially due to the difficulty to discriminate between pulses and gravity field parameters.

\section{The GRACE baseline}

The so-called GRACE baseline in its original sense stands for the expected accuracy of the GRACE-derived monthly gravity fields, established prior to the launch of the mission. The GRACE baseline is expressed in difference degree amplitudes. It is based on covariance studies and on "endto-end" simulations using realistic error estimates including white and colored noise for all measurement sensors and the non-modeled parts of the force field. Crude estimates may be found in Thomas (1999), a refined analysis, probably the origin of the official "GRACE baseline", in Kim (2000). The latter GRACE performance estimate is based on a realistic simulation study with three kinds of parameters (a) orbit parameters (initial conditions and empirical dynamic parameters, constant and once-per-revolution periodic terms), (b) gravity field parameters, and (c) so-called kinematic empirical parameters (offset and drift, once-perrevolution terms for the range and the range-rate observables, where the amplitudes are linear functions of time). Each set of parameters of type (c) and of dynamic empirical parameters is valid for a time interval varying between $1 / 2$ and 1 revolution period). This processing scheme is still in use today at some of the GRACE analysis centers, however, without the once-per-revolution terms with amplitudes linearly growing with time, see Meyer et al. (2009). The CMA differs in two essential aspects from the above baseline estimates:

1. The CMA admits one parameter of type (c) for each time interval of uninterrupted K-Band Level 1a ranges, namely the range offset. No parameters of type (c) are allowed when analyzing range-rates. 
Table 4 CMA processing options for K-Band analysis

\begin{tabular}{llll}
\hline Solution & $\sigma_{\text {apr }} / \sigma_{\text {post }}$ & Rel. constraint & Characteristics \\
\hline GRR14UP30 & $0.2 / 0.276$ & - & Pulse spacing of 30 min, $R R$ \\
GRR14UP15 & $0.2 / 0.249$ & - & Pulse spacing of 15 min, $R R$ \\
GRR14UP051612 & $0.2 / 0.322$ & $1.0 \times 10^{-8} \mathrm{~m} / \mathrm{s}$ & Pulse spacing of 5 min, $R R$ \\
GRG34UP30 & $0.7 / 7.40$ & - & Pulse spacing of 30 min, $R$ \\
GRG34UP15 & $0.7 / 3.53$ & - & Pulse spacing of 15 min, $R$ \\
GRG34UP051612 & $0.7 / 1.87$ & $1.0 \times 10^{-8} \mathrm{~m} / \mathrm{s}$ & Pulse spacing of 5 min, $R$ \\
\hline
\end{tabular}
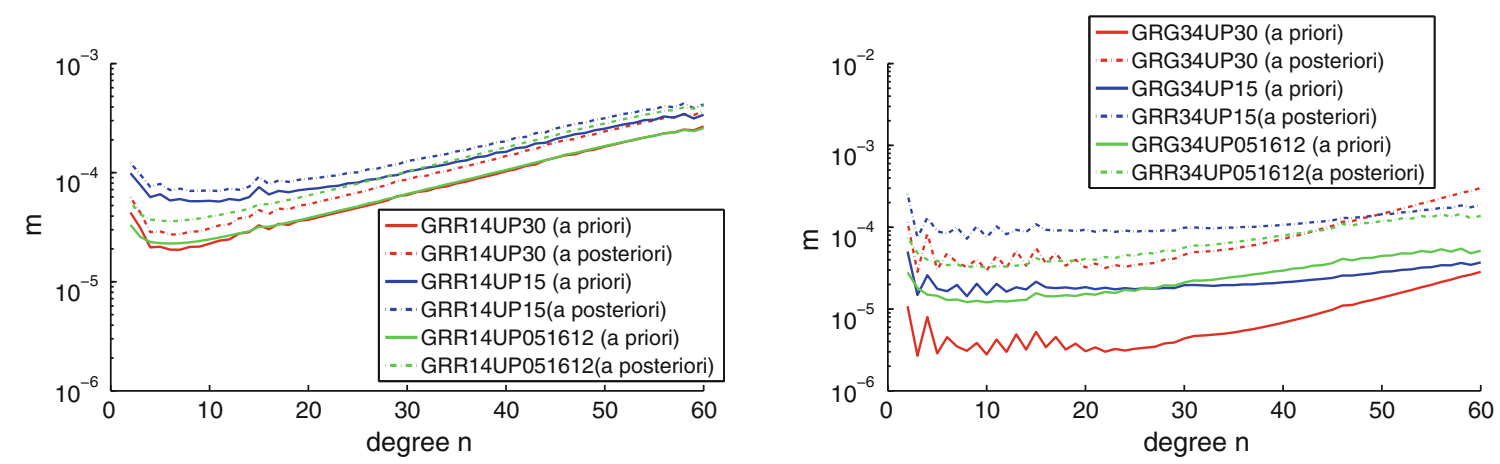

Fig. 21 GRACE baselines, expressed in error degree amplitudes, for the solutions in Table 4; 30 days, geoid heights; left range-rate, right range

2. The CMA allows for the estimation of (constrained) pseudo-stochastic parameters, which have the capability to absorb the non-modeled parts of the force field.

From our perspective there is no justification for introducing other (than offset) parameters of type (c). Kim (2000) justifies the parameters of type (c) in essence with the white and colored noise present in the accelerometer measurements or in the background models. The parameters of type (c) thus play a similar role as the pseudo-stochastic parameters in the CMA. Both error sources do exist, but from our perspective they should be dealt with on the level they occur, i.e., they should be absorbed by empirical dynamic parameters or by pseudo-stochastic parameters. The constant accelerations in $R, S, W$, the once-per-revolution terms, and the pulses or the piecewise constant accelerations assume this role in the CMA.

For these reasons it seems appropriate to generate CMAspecific "GRACE baselines", also in terms of error degree amplitudes, for the more important processing strategies discussed previously. Two kinds of "baselines" are provided: Those based on the inverted NEQ-matrix multiplied by the a priori RMS of the observable and those based on the estimated covariance matrix.

As the classical GRACE baseline is usually referred to data spans of one month and is expressed in geoidal heights, the CMA-specific GRACE baselines are also provided in geoidal heights (in $\mathrm{m}$ ) referring to 30 days of data, by multiplying the dimensionless error degree amplitudes by $a_{e} \times \sqrt{50 / 30} \approx 1.291 \times a_{e}$, where $a_{e}=6,378,137 \mathrm{~m}$ is the equatorial radius of the Earth. This re-scaling gives slightly too optimistic values, as the effect of the improved geographical coverage from 30 to 50 days is not taken into account.

Table 4 summarizes the six solutions, for which "baselines" are provided. Three of them are based on range-rate (without correlations) and three on range (without correlations). Two solutions out of the three in each group are contiguous, but otherwise unconstrained short-arc solutions, the other one is based on constrained pulses (separated by $5 \mathrm{~min}$ ). The constraints may also be found in Table 4. The baselines are of a similar order of magnitude for range-rate and range-if the covariance matrix is used to calculate the error degree amplitudes (dash-dot curves). If the RMS a priori is used for scaling the inverted NEQ matrix, the baselines which are derived from the range observable and which are based on (comparatively) few pseudo-stochastic parameters promise to be much better than the corresponding baseline using the covariance matrix. This statement is best seen in Fig. 21, red curves. The big difference of course only proves that

- there are either substantial non-modeled systematics in the force field not captured by the accelerometers (e.g., errors in the background models describing temporal gravity field variations like tidal effects, de-aliasing products);

- or that the accelerometers do not perform as they should; 

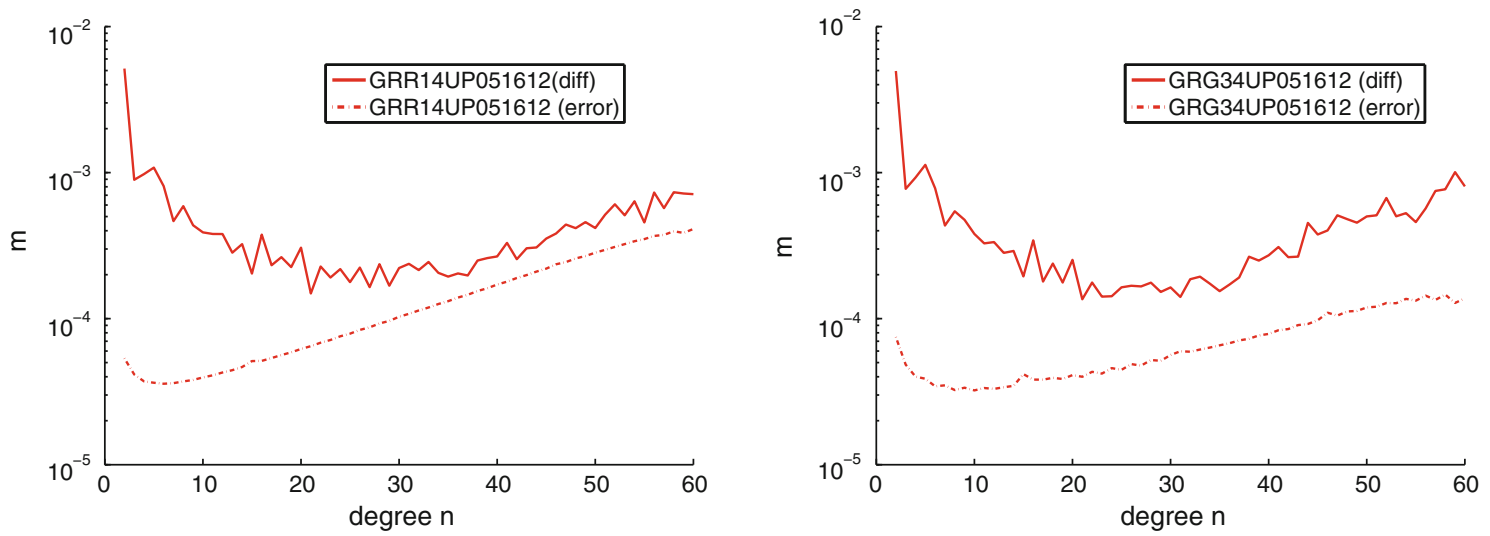

Fig. 22 GRACE baselines, expressed in error degree amplitudes, and corresponding difference degree amplitude w.r.t. AIUB-GRACE02S, left range-rate, right range

- or that additional information needed to calculate the center-of-mass to center-of-mass distance between the two satellites is substantially wrong;

- or that the reduction of the observations (from the raw $\mathrm{K}$-Band measurements to the Level 1a ranges) is not correct.

Figure 22 shows the difference degree amplitudes of the 30 days 5 min constrained solution (range-rates (left), ranges (right)) w.r.t. the biennial solution AIUB-GRACE02S. One clearly sees a big discrepancy towards the low degrees, whereas the higher difference degree amplitudes follow the curve for the error degree amplitudes quite well. There still is a discrepancy corresponding to a factor varying between 3 and 10 between the achieved and the expected accuracy for the corresponding range solution in Fig. 22 (right). The missing time-varying signal in the mean field model (e.g., due to hydrology) may be responsible for some of the discrepancies in the region of the low degree harmonics.

\section{Summary and conclusions}

The CMA was applied to 50 days of GRACE data, namely, to DOY 50-99/2007. Orbit and gravity field determination were considered. The gravity fields were always based on the full set of 50 days of data in 2007. The solutions differ in the underlying observables, in the parametrization, and in the constraints imposed on the estimated orbit and/or pseudostochastic parameters. No constraints were imposed on the estimated gravity field parameters. Only the spherical harmonics within the limits $2 \leq n \leq 60$ were determined, the terms $61 \leq n \leq 150$ were taken over from the solution AIUB-GRACE02Sp, which is based on the entire year 2007 of GRACE data.
Orbit determination was studied in Sect. 4. The RMS error a posteriori of the GPS L1 phase observations was found to be about $\sigma_{p h} \approx 2 \mathrm{~mm}$ in an orbit determination experiment using only the kinematic positions (together with the epochspecific weight matrix) as pseudo-observations. The RMS a posteriori of the K-Band range-rate observable was established by a "K-Band only" analysis as $\sigma_{k b d, r r} \approx 0.2 \mu \mathrm{m} / \mathrm{s}$, implying that the correct weight ratio to combine the K-Band range-rate and GPS NEQs is $\sigma_{p h}^{2} / \sigma_{k b d, r r}^{2} \approx 10^{8} \mathrm{~s}^{2}$. This ratio was used for orbit and gravity field determination in our analyses.

It is an open issue to be addressed in future why the best consistency of gravity fields determined by the CMA with those of other GRACE analysis teams is achieved with a weight ratio $\sigma_{p h}^{2} / \sigma_{k b d, r r}^{2} \approx 10^{10} \mathrm{~s}^{2}$, which considerably deweights the GPS contribution.

Standard procedures for gravity field estimation were discussed in Sect. 5. GPS-only, K-Band-only, and combined GPS and K-Band gravity fields were produced. All K-Band contributions used the option $R R$ in Table 3. Pulses in $R, S$, and $W$ with a spacing of either 30 , or 15 , or $5 \mathrm{~min}$ were set up in different solutions. No or only weak constraints (5 min solution) were imposed on the pulses. The main findings were:

- GPS-only gravity fields up to a maximum degree of $n_{\max } \approx 60$ may be determined using a data span of 50 days-adopting the terms above $n=60$ from an a priori solution of good quality.

- The sectorial terms are much better determined by GPS than the zonal terms. Actually, the sectorial terms are determined with GPS with an accuracy comparable to that of K-Band-only solutions.

- K-Band-only solutions are based on an a priori orbit established with K-Band and GPS (in this sense the attribute 
"K-Band-only" is not correct). All terms, except those of low degree $n \lesssim 5$, are established with high accuracy.

In Sect. 6 it was shown that the $R R$-solution (Table 3) with strongly constrained $5 \mathrm{~min}$ pulses is of comparable quality as the 30 min pulse solution without constraints.

The impact of accelerometer data on gravity field determination using option $R R$ (Table 3) was studied in Sect. 7. The main findings are:

- Gravity fields of good quality may be established even without using acclerometer data.

- The difference degree amplitudes w.r.t. AIUB-GRACE $02 \mathrm{~S}$ when using accelerometer data improve by a factor of about 1-2.

- The $S$ component of accelerometer data has the largest impact on the K-Band observables.

Alternatives to option $R R$ (Table 3 ) were studied in Sect. 8. The key findings are:

- Options $R R$ and $R D$ (Table 3) are equivalent, provided the statistical information is chosen according to Eqs. (7, 8, 12).

- Options $R$ and $R C$ (Table 3 ) are equivalent, provided the statistical information is chosen according to Eqs. $(9,11$, 12).

- Options $R R C$ and $R C$, thus also $R$, (Table 3 ) are equivalent, provided the statistical information is chosen according to Eqs. $(9,11,12)$

In Sect. 9 the residuals of range and range-rate solutions were analyzed. Systematic effects dominate the range solution. It was shown that the use of accelerometer data has a much larger impact on range residuals than on range-rate residuals. The overall fit improves roughly by a factor of 2 when using the accelerometer data (the gain is much smaller for range-rate residuals). The residuals of ranges and rangerates were spectrally analyzed for the entire interval of 50 days. For periods $P<15 \mathrm{~min}$ the spectrum is richly populated with terms of amplitudes of the order of $10^{-8} \mathrm{~m} / \mathrm{s}$ for range-rate, of the order of $10^{-6} \mathrm{~m}$ for ranges - indicating the presence of systematic accelerations not absorbed by the accelerometers.

In Sect. 10 we eventually produced a solution based on ranges (option $R$, Table 3 ), which is of comparable quality as the best range-rate solutions presented here. Unfortunately, many constrained pulses spaced by $5 \mathrm{~min}$ had to be set up, what weakened the much higher potential of the range solutions to determine the gravity field.

In Sect. 11 the GRACE baseline, as achievable currently by the CMA, was established. When taking into account the non-modeled and/or the stochastic part of the force-field the discrepancy of the difference degree amplitudes w.r.t. AIUBGRACE02 and the error degree amplitudes are small for degrees $n \gtrsim 20$ for option $R R$ (Table 3 ), somewhat larger for options $R$ and $R C$. The error degree amplitudes are clearly too optimistic for low degrees $n \lesssim 10$. More work, in particular concerning the GPS contribution, is required.

Acknowledgements This work was supported by the Swiss National Science Foundation (project "gravity field determination using positions of low Earth orbiters established with the GPS" with number 200020 117908) and by the Institute for Advanced Study (IAS) of the Technische Universität München. Both contributions are gratefully acknowledged.

\section{References}

Bettadpur S (2007) GRACE 327-720 (CSR-GR-03-02) Gravity recovery and climate experiment product specification document (Rev 4.5, February 20, 2007), Center for Space Research, the University of Texas at Austin

Beutler G, A Jäggi, U Meyer, L Mervart (2010) The celestial mechanics approach: theoretical foundations. J Geod. doi:10. 1007/s00190-010-0401-7

Dach R, Hugentobler U, Meindl M, Fridez P (eds) (2007) The Bernese GPS Software Version 5.0, Astronomical Institute, University of Bern

Descartes R (1637) Discours de la methode Pour bien conduire sa raison, \& chercher la verité dans les sciences, Plus la dioptriuve, les meteores, et la geometrie, Qui sont des essais de cete Methode. A Leyde, De l'Imprimerie de Ian Maire MDCXXXVII

Ditmar P, Klees R, Liu X (2007) Frequency-dependent data weighting in global gravity field modeling from satellite data contaminated by non-stationary noise. J Geod 81: 81-96. doi:10.1007/ s00190-006-0074-4

Förste C, Flechtner F, Schmidt R, Stubenvoll R, Rothacher M, Kusche J, Neumayer KH, Biancale R, Lemoine JM, Barthelmes F, Bruinsma S, König R, Meyer U (2008) EIGEN-GL05C - a new global combined high-resolution GRACE-based gravity field model of the GFZ-GRGS cooperation. Geophysical Research Abstracts, vol 10

Jäggi A (2007) Pseudo-stochastic orbit modeling of low earth satellites using the Global Positioning System. Geodätisch-geophysikalische Arbeiten in der Schweiz, Vol 73, Schweizerische Geodätische Kommission, Institut für Geodäsie und Photogrammetrie, Eidg. Technische Hochschule, Zürich

Jäggi A, Beutler G, Prange L, Meyer U, Mervart L, Dach R, Rummel R, Gruber T (2009) Gravity field determination at AIUB: current activities. EGU General Assembly 2009, EGU2009-8714

Jäggi A, Beutler G, Meyer U, Mervart L, Prange L, Dach R (2010) AIUB-GRACE02S-status of GRACE gravity field recovery using the celestial mechanics approach. International Association of Geodesy Symposium (accepted for publication)

Kim J (2000) Simulation study of a low-low satellite-to-satellite tracking mission. Ph D Dissertation, The University of Texas at Austin, May 2000

Lemoine FG, Smith DE, Kunz L, Smith R, Pavlis NK, Klosko SM, Chinn DS, Torrence MH, Willamson RG, Cox CM, Rachlin KE, Wang YM, Kenyon SC, Salman R, Trimmer R, Rapp RH, Nerem RS (1997) The development of the NASA GSFC and NIMA Joint Geopotential Model. In: Segawa J, Fujimoto H, Okubo S (eds) IAG symposia gravity, geoid and marine geodesy. Springer, Berlin, pp 461-469 
Meyer U, Flechtner F, Schmidt R, Frommknecht B (2009) A simulation study discussing the GRACE baseline accuracy. International association of geodesy symposium (accepted for publication)

Prange L, Jäggi A, Beutler G, Mervart L, Dach R (2009) Gravity field determination at the AIUB - the Celestial mechanics approach. In: Sideris MG (ed) Observing our changing Earth. Springer, Berlin, pp 353-362. doi:10.1007/978-3-540-85426-5-42

Prange L, Jäggi A, Bock H, Dach R (2010) The AIUB-CHAMP02S and the influence of GNSS model changes on gravity field recovery using spaceborne GPS. Adv Space Res 45(2):215-224. doi:10. 1016/j.asr.2009.09.020
Thomas J.B. (1999) An analysis of gravity-field estimation based on intersatellite dual-1-way biased ranging. JPL Publication 98-15 http://podaac.jpl.nasa.gov/pub/grace/doc/newsletters/ GRACE_SDS_NL_0401.pdf

Touboul P, Willemenot E, Foulon B, Josselin V (1999) Accelerometers for CHAMP, GRACE and GOCE space missions: synergy and evolution. Boll Geof Teor Appl 40:321-327 